\title{
Situationsbezug und die Stadien/Individuen-Distinktion bei Kopula-Prädikativ-Konstruktionen
}

\author{
Claudia Maienborn \\ Humboldt-Universität zu Berlin \\ c.maienborn@rz.hu-berlin.de
}

\begin{abstract}
Within the Davidsonian paradigm copula-predicative constructions are commonly assumed to involve a state argument. Its source is taken to be either the copula be (cf. e.g. Bierwisch 1988) or the predicative (cf. the ongoing stage level/individual level debate). Yet, a critical examination of copulapredicative constructions in contexts that call for Davidsonian arguments (locative modifiers, manner adverbials, perception verbs, etc.) reveals that they do not behave as expected. In fact, the data examined here do not support the assumption that copula-predicative constructions are equipped with a Davidsonian argument nor is there any evidence for a grammatically reflected distinction between temporary and permanent properties. The present paper argues alternatively for a grammatical distinction between states like sit, stand, sleep, wait, live and statives like resemble, know, hate, cost which is invoked by the presence or absence of a Davidsonian argument. Copula-predicative constructions are shown to belong uniformly to the class of statives. The acceptability differences of copula-predicative constructions in combination with locative modifiers are accounted for pragmatically on the basis of conversational implicatures.
\end{abstract}

\section{Situationsbezug von Kopula-Prädikativ-Konstruktionen}

Seit Davidsons (1967) epochemachendem Aufsatz zur Semantik von Handlungssätzen haben Situationen ${ }^{1}$ ihren Siegeszug durch die Verbsemantik angetreten. Es scheint daher naheliegend, dieses Instrumentarium auch auf dem Gebiet der Kopula-Prädikativ-Konstruktionen einzusetzen. Nach gängiger Auffassung innerhalb des Davidsonschen Paradigmas bezeichnet die Kopula sein zusammen mit dem Prädikativ eine Situation, bzw. genauer: einen Zustand; s. z.B. Bierwisch (1988), Parsons (1990, 1995), Kamp \& Reyle (1993). ${ }^{2}$ Kopula plus Prädikativ verhalten sich demzufolge wie reguläre Zustandsverben vom Typ sitzen, stehen, schlafen. (Mein Verständnis von regulären Zustandsverben werde ich in Abschnitt 2.3 präzisieren.) In der Frage, wodurch ein entsprechendes Situations- bzw.

\footnotetext{
* Dieser Beitrag ist im Rahmen des von der DFG am Zentrum für Allgemeine Sprachwissenschaft in Berlin geförderten Projekts "Schnittstellen der Semantik: Prädikativkonstruktionen" entstanden. Für anregende Diskussionen, Hinweise und Kritik danke ich Manfred Bierwisch, Reinhard Blutner, Hannes Dölling, Annette Fischer, Ljudmila Geist, Gerhard Jäger, Ewald Lang, Renate Musan, Cristina Schmitt, Renate Steinitz und Ilse Zimmermann.

${ }^{1}$ Ich verwende den Terminus "Situation" als Oberbegriff für Ereignisse, Prozesse und Zustände. In der neueren Literatur findet sich hierfür auch die Bezeichnung "spatiotemporal location" z.B. bei Kratzer (1989) oder die Bezugnahme auf ein "Davidsonian argument" z.B. bei Chierchia (1995). Bach (1986) schlägt als Oberbegriff "Eventualität" vor, legt dabei jedoch ein weiter gefaßtes Verständnis von Zuständen zugrunde, als es in der vorliegenden Arbeit vertreten wird; s. hierzu Abschnitt 2.3.

2 Ich werde im Rahmen dieser Arbeit nicht auf die vornehmlich in der Tradition der sprachanalytischen Philosophie stehenden Ansätze eingehen, die die Frage des Situations- bzw. Zeitbezugs der Kopula nicht thematisieren; vgl. hierzu den Überblick in Dölling (1997). Ebensowenig werde ich die Frage der Unterscheidung einer prädizierenden von einer identifizierenden Funktion der Kopula berühren; s. wiederum Dölling (1997).
} 
Kopula wird s dadurch bestimmt, daß das Prädikat Q auf das Individuum x zutrifft. Das Situationsargument s steht im weiteren u.a. für die temporale Einordnung bereit und wird in einem späteren Stadium der Bedeutungskomposition existentiell gebunden; s. Maienborn (1998). Für den Satz in (2.a) ergibt sich (bei Annahme einer VP-internen Subjektposition) als VP-Repräsentation die SF in (2.b). Die VP bezeichnet damit die Menge von Situationen in denen Heidi müde ist. Unter Vernachlässigung von Tempus, Verbmodus usw, erhalten wir für den Satz die SF in (2.c).

(2) a. Heidi ist müde.

b. VP: $\lambda$ s [s INST [TIRED (Heidi)]]

c. CP: $\exists$ s [s INST [TIRED (Heidi)]]

Betrachten wir zum Vergleich die SF eines Vollverbs wie schlafen in (3). Ganz analog zum Fall der Kopula-Prädikativ-Konstruktion erhält der Satz (4.a) die SFs in (4.b/c).

(3) schlafen: $\lambda \times \lambda s$ [s INST [SLEEP (x)]]

(4) a. Heidi schläft.

b. VP: $\lambda s$ [s INST [SLEEP (Heidi)]]

c. CP: $\exists$ s [s INST [SLEEP (Heidi)]]

Der hier vorgestellte Ansatz macht die Vorhersage, daß Kopula-Prädikativ-Konstruktionen sich genauso verhalten wie andere VPn, die Zustände denotieren. Die Grammatik macht demnach keinen Unterschied zwischen dem (zeitweiligen) Vorliegen einer Eigenschaft bei einem Individuum und dem Vorliegen eines Zustandes, an dem ein oder mehrere Individuen als Partizipanten beteiligt sind. Ausdruck hierfür ist die Annahme eines Situationsreferenten in der Semantik der Kopula.

\subsection{Quelle für das Situationsargument: Prädikativ}

Mit Kratzer (1989) liegt ein im Rahmen der Stadien/Individuen-Diskussion vielfach aufgegriffener Vorschlag vor, Verben und Prädikative, die temporäre Eigenschaften bezeichnen (sog. Stadienprädikate), mit einem Situationsargument auszurüsten. Die Anwesenheit eines Situationsarguments unterscheidet Stadienprädikate ("stage level predicates"; kurz: SLP) wie in (5) von sog. Individuenprädikaten ("individual level predicates"; kurz: ILP) wie in (6). Letztere bezeichnen (tendentiell) permanente Eigenschaften von Individuen. ${ }^{3}$

(5) Stadienprädikate (SLP):

Adjektive: müde, betrunken, hungrig, aufgeregt ...4

Verben: schlafen, warten, laufen, bezahlen, ankommen, ...

$\cdots$

${ }^{3}$ Die Unterscheidung von Stadien- und Individuenprädikaten geht auf Carlson (1977) zurück. Carlson betrachtet die Stadien/Individuen-Distinktion als ontologische Unterscheidung und nimmt neben Individuen auch Stadien (raumzeitliche Ausschnitte von Individuen) als Entitäten an. SLP sind folglich Prädikate über Stadien, ILP Prädikate über Individuen. Kratzers Ansatz hingegen verzichtet auf derartige ontologische Annahmen und verlagert den Unterschied in die Argumentstruktur der Prädikate.

${ }^{4}$ Im weiteren klammere ich die oft als Paradebeispiele für SLP angeführten Adjektive verfïgbar und sichtbar (bzw. available und visible) aus. Die insbesondere im Bereich der Subjekteigenschaften als SLP-typisch dingfest gemachten Charakteristika scheinen mir eher auf Besonderheiten dieser Adjektive zu beruhen; s. Maienborn (1996), Jäger (1999). So hält beispielsweise die ausgiebig diskutierte und variantenreich unterlegte Feststellung, ein Satz mit einem SLP wie in (i) lasse sowohl eine generische als auch eine existentielle Interpretation zu, wohingegen im Falle eines ILP wie in (ii) nur die generische Interpretation zur Verfügung stehe, einer Ubertragung auf andere SLP-Fälle wie in (iii) nicht stand. Die Sätze in (iii) erlauben nur die generische Interpretation.

(i) Feuerwehmänner sind verfügbar.

(ii) Feuerwehrmänner sind selbstlos.

(existentielle \& generische Interpretation)

(nur generische Interpretation)

(iii) Feuerwehrmänner sind hungrig/müde/aufgekratzt. (nur generische Interpretation)

Im Falle von verfügbar, sichtbar usw. schiene es mir sinnvoll, zunächst einmal die interne Semantik dieser deverbalen Adjektive genauer zu untersuchen. (Dies aber ist nicht Gegenstand der vorliegenden Untersuchung.) 
(6) Individuenprädikate (ILP):

Adjektive: intelligent, blond, selbstlos, ...

Nomen: Vegetarierin, Förster, Primzahl, ...

Verben: wissen, lieben, ähneln, heißen, ...

...

In (7) liegt ein Stadienprädikat vor, in (8) ein Indidviduenprädikat.

(7) a. Heidi ist müde.

b. $\exists$ s [TIRED (Heidi, s)]

(8) a. Heidi ist Vegetarierin.

b. VEGETARJAN (Heidi)

Die syntaktischen und semantischen Phänomene, die im Laufe der Zeit mit der Stadien/IndividuenDistinktion in Verbindung gebracht wurden, sind Legion; s. die Zusammenstellung und Kommentierung in Jäger (1999). Von Beginn an sahen sich Stadien/Individuen-Ansätze Kritik u.a. an der je nach Bedarf recht freizügig gehandhabten Umkategorisierung von SLP in ILP und retour ausgesetzt. In letzter Zeit mehren sich die Hinweise darauf, daß (a) unter dem Etikett "Stadien/Individuen-Distinktion" ein Bündel von durchaus unterschiedlichen Phänomenen zusammengefaßt wurde; s. Higginbotham \& Ramchand (1996), Jäger (1999) und (b) für die angepeilten Kontraste vielmehr TopikKommentar-Gliederung und Generizität ausschlaggebend sind; s. Chierchia (1995), Krifka et al. (1995), Jäger (1997). Im Zentrum meiner weiteren Überlegungen steht deshalb allein die Frage nach einer grammatisch reflektierten Unterscheidung von temporären und permanenten Eigenschaften. Interessanterweise ist diese Frage der semantisch/ontologischen Fundierung des Stadien/IndividuenKontrastes in der gesamten Literatur bislang deutlich vernachlässigt worden.

Als eines der zentralen Kriterien für die Unterscheidung von SLP und ILP gilt die Kombinierbarkeit mit lokalen Modifikatoren; s. z.B. Kratzer (1989: 126ff), Chierchia (1995: 178f). Der generelle Befund lautet: SLP sind mit lokalen Modifikatoren kombinierbar, ILP hingegen nicht. Als Grundlage dienen Kontraste wie die in (9).

(9) a. Heidi war im Auto müde/betrunken/hungrig.

b. ??Heidi war im Auto Vegetarierin/intelligent/blond.

Der hier skizzierte Rahmen liefert eine naheliegende Erklärung für dieses Verhalten: Mit Kratzers Situationsargument bieten die SLP in (9.a) eine geeignete Anschlußmöglichkeit für ein Lokaladverbial. Bei ILP ist dieser Weg versperrt. Die Konstruktionen in (9.b) sind folglich abweichend. ${ }^{5}$ Chierchia (1995: 178) merkt hierzu an: »Intuitively, it is as if i-level predicates were, so to speak, unlocated. If one is intelligent, one is intelligent nowhere in particular. S-level predicates, on the other hand, are located in space. « Temporäre Eigenschaften sind demnach räumlich situiert, permanente Eigenschaften hingegen ortsungebunden.

Zulässige Kombinationen von ILP mit lokalen Modifikatoren wie in (10) werden im Stadien/Individuen-Paradigma darauf zurückgeführt, daß hier das Lokaladverbial zur Beschränkung einer Quantorendomäne dient. Für den Satz (10.a) wird auf der syntaktischen Ebene der Logischen Form die Struktur in (10.b) angesetzt; s. z.B. Kratzer (1989: 140), Krifka et al. (1995: 26f), Chierchia (1995: 178). Satz (10.a) erhält demzufolge die Interpretation, daß für viele Schwäne in Australien gilt, daß sie schwarz sind.

5 Die Bewertung von Sätzen des Typs (9.b) mit 2 Fragezeichen findet sich z.B. bei Chierchia (1995: 178). Da ich in Abschnitt 2 einige der Prämissen für die Datenbeurteilung in (9) entkräften möchte, will ich die Legitimation und Aussagekraft dieser Kennzeichnung hier nicht weiter kommentieren. 
(10) a. In Australien sind viele Schwäne schwarz.

b. LF: Viele $x\left[_{\mathrm{RC}} \operatorname{Schwan}(\mathrm{x}) \& \text { in Australien (x)] [ }{ }_{\mathrm{NS}} \operatorname{schwarz}(\mathrm{x})\right]^{6}$

In den letzten Jahren findet es zunehmend Verbreitung, sämtliche Prädikate mit einem Situationsargument auszustatten. Im Rahmen ihrer Überlegungen zur Stadien/Individuen-Distinktion schlagen dies z.B. Chierchia (1995) und Higginbotham \& Ramchand (1996) vor. Ohne Bezug auf den SLP/ILP-Kontrast gehen beispielsweise Parsons (1990), Higginbotham (1994) und Jacobs (1995) diesen Weg.

Die hier vorgestellten Ansätze stimmen darin überein, daß Kopula-Prädikativ-Konstruktionen vom Typ (2.a) bzw. (7.a) mit einem Situationsargument ausgestattet sind und sich damit nicht von Sätzen mit einem Zustandsverb vom Typ (4.a) unterscheiden. Wenn eine grammatisch relevante Unterscheidung vorgenommen wird, dann zwischen Kopula-Prädikativ-Konstruktionen vom Typ (2.a)/(7.a) und (8.a), d.h. zwischen dem Auftreten temporärer Eigenschaften bei Individuen einerseits und dem Vorliegen permanenter Individueneigenschaften andererseits. Soweit die Ausgangslage.

\section{Evidenzen gegen die Präsenz eines Situationsarguments in Kopula- Prädikativ-Konstruktionen}

Im folgenden will ich Argumente gegen die in Abschnitt 1 geschilderte Auffassung vom Situationsbezug bei Kopula-Prädikativ-Konstruktionen aufführen. Dabei werde ich eine Reihe von Tests anwenden, die die Präsenz eines Situationsarguments anzeigen. Hierzu zählen insbesondere die Kombination mit lokalen (und anderen) Modifikatoren und die Kombination mit Wahrnehmungsverben. Es wird sich zeigen, daß Kopula-Prädikativ-Konstruktionen bei diesen Tests nicht das für Situationsausdrücke prognostizierte Verhalten an den Tag legen.

\subsection{Lokale Modifikatoren}

Beginnen wir bei der Kombination von Kopula-Prädikativ-Konstruktionen mit lokalen Modifikatoren. Seit Davidson (1967) und Vendler (1967) hat sich als Mindestanforderung an eine ontologische Bestimmung von Situationen die Charakterisierung als raumzeitliche Entitäten eingebürgert und bewährt. Situationen sind Entitäten, die in Zeit und Raum lokalisierbar sind; s. auch Ehrich (1991). Situationsausdrücke sind folglich durch Lokaladverbiale modifizierbar.

Wie steht es damit nun bei Kopula-Prädikativ-Ausdrücken? Um diese Frage zu beantworten, ist es zunächst wichtig, verschiedene Arten lokaler Modifikation zu unterscheiden. Ich habe in Maienborn (1996, 1998) für die Unterscheidung von drei Typen lokaler Modifikatoren von Verbalprojektionen argumentiert. Diese zeichnen sich syntaktisch durch unterschiedliche Basispositionen aus. Mit der jeweiligen Plazierung geht semantisch ein eigener Beitrag zur Bedeutungskomposition einher. ${ }^{7} \mathrm{Im}$ Beispielsatz (11) ist jeder Modifikator-Typ vertreten.

(11) In den Anden werden Schafe vom Pfarrer vor der Kirche an den Ohren gebrandmarkt.

I

rahmensetzend $\sqrt{3}$

situationsextern situationsintern

\footnotetext{
${ }^{6}$ Der Ansatz einer dreigeteilten logischen Repräsentation bestehend aus (nicht-selektivem) Quantor, restrictive clause (RC) und nuclear scope (NS) geht auf Lewis (1975), Kamp (1981) und Heim (1982) zurück. Im Stadien/IndividuenParadigma wird diese Analyse häufig mit Diesings (1992, 1992a) sogenannter "Mapping Hypothese" kombiniert, derzufolge VP-Material in den nuclear scope eingeht, VP-externes Material in den restrictive clause.

7 Die aus der unterschiedlichen syntaktischen Plazierung resultierenden prosodischen Differenzen bei lokalen Modifikatoren werde ich hier nicht eigens thematisieren; s. aber Maienborn $(1996,1998)$.
} 
Das Lokaladverbial in den Anden gehört zur Klasse der rahmensetzenden Modifikatoren. Diese haben eine syntaktische Basisposition an der linken Peripherie des Satzes. (In Maienborn (1998) schlage ich vor, diese Modifikatoren als Adjunkte an TopP zu analysieren.) Ihr semantischer Beitrag besteht in der Setzung eines lokalen Rahmens für die gesamte Proposition. Das Lokaladverbial vor der Kirche ist ein Vertreter der situationsexternen Modifikatoren. Diese sind an der VP-Peripherie angesiedelt, und ihr semantischer Beitrag besteht in der räumlichen Situierung der durch die VP bezeichneten Situation. Das Lokaladverbial an den Ohren schließlich ist ein situationsinterner Modifikator. Diese nehmen eine Basisposition in unmittelbarer Nähe des Verbs ein und liefern zusätzliche räumliche Information über den internen Aufbau der Situation.

Im Unterschied zu situationsexternen und situationsinternen Modifikatoren beschränken rahmensetzende Modifikatoren nicht den Situationsreferenten des Verbs, sondern sie liefern eine semantisch unterbestimmte Beschränkung für die Gesamtproposition. ${ }^{8}$ Die genaue Festlegung der Beschränkung erfolgt pragmatisch. Damit zeichnen sich rahmensetzende Modifikatoren durch ein ganzes Spektrum potentieller Äußerungsbedeutungen aus; zu den Einzelheiten einer semantischen und pragmatischen Analyse s. Maienborn (1998). An dieser Stelle möge ein Beispiel zur Veranschaulichung genügen:

(12) In Chile genießt Pinochet diplomatische Immunität.

Für (12) bieten sich je nach kontextuellem Hintergrund mindestens drei Ausbuchstabierungen der räumlichen Rahmensetzung an. Das Lokaladverbial kann zur Beschränkung des Geltungsbereichs diplomatischer Immunität herangezogen werden. Dies ist ohne weitere Kontextvorgaben sicherlich die präferierte Lesart für (12). Daneben besteht für rahmensetzende Modifikatoren grundsätzlich die Möglichkeit einer epistemischen Interpretation. Im Falle von (12) besagt diese, daß nach Auffassung einer relevanten Gruppe von Personen in Chile Pinochet (möglicherweise weltweit) diplomatische Immunität genießt. ${ }^{9}$ Schließlich erlauben rahmensetzende Modifikatoren auch temporale bzw. konditionale Interpretationen. Die entsprechende Lesart für (12) besagt, daß Pinochet, immer dann, wenn er sich in Chile aufhält, diplomatische Immunität genießt.

Um festzustellen, ob wir es mit einem rahmensetzenden oder einem der beiden situationsbezogenen Modifikatoren zu tun haben, empfiehlt sich der Blick auf das Inferenzverhalten. Anders als die situationsbezogenen Modifikatortypen unterstützen rahmensetzende Modifikatoren nicht das klassische Inferenzmuster, das einer der zentralen Anlässe für die Einführung von Situationsreferenten bei Davidson war; s. (13) - (15).

(13) a. Der Pfarrer hat vor der Kirche ein Schaf gebrandmarkt.

situationsexterner Modifikator b. $\rightarrow$ Der Pfarrer hat ein Schaf gebrandmarkt.

(14) a. Der Pfarrer hat ein Schaf am Ohr gebrandmarkt. situationsinterner Modifikator b. $\rightarrow$ Der Pfarrer hat ein Schaf gebrandmarkt.

(15) a. In den Anden werden Schafe vom Pfarrer gebrandmarkt. b. $\rightarrow$ Schafe werden vom Pfarrer gebrandmarkt.

rahmensetzender Modifikator

${ }^{8}$ Neben den für unsere Frage zentralen Lokaladverbialen treten auch andere Adverbiale als rahmensetzende Modifikatoren auf; s. (i) - (iii). Wir haben es hier also nicht mit einer Besonderheit des räumlichen Ausdrucksinventars zu tun.

(i) Mit der Bahn dauert die Fahrt zwei Stunden.

(ii) Nach Geschäftsschluß ist die Innenstadt menschenleer.

(iii) Für den Schiedsrichter hat der Ball die Torlinie passiert.

9 Vgl. das in (i) aufgeführte Zitat von Harald Juhnke aus dem Jahr 1998, dessen Äußerungsbedeutung auf der epistemischen Interpretation des rahmensetzenden Modifikators beruht. (i) besagt demnach, daß die Menschen in Deutschland der Auffassung sind, Harald Juhnke sei weltberühmt.

(i) In Deutschland bin ich weltberühmt. 
c. In Chile genießt Pinochet diplomatische Immunität

d. $\rightarrow$ Pinochet genießt diplomatische Immunität.

Allein im Falle der temporalen Interpretation eines rahmensetzenden Modifikators ist das oben angegebene Inferenzmuster gültig. Wenn wir für (16.a) die temporale Lesart zugrundelegen, derzufolge Pinochet zu der Zeit, als er sich in Chile aufhielt, diplomatische Immunität besaß, dann können wir auf (16.b) schließen. Für die übrigen Äußerungsbedeutungen von (16.a), etwa die epistemische Interpretation, gilt dies jedoch weiterhin nicht.

(16) a. In Chile genoß Pinochet diplomatische Immunität.

b. $\rightarrow$ Pinochet genoß diplomatische Immunität. rahmensetzender Modifikator bei temporaler Interpretation

Angesichts des Inferenzverhaltens der Modifikatoren in (16) und (13) mag der Eindruck entstehen, die temporale Lesart rahmensetzender Modifikatoren sei identisch mit der Interpretation situationsexterner Modifikatoren. Dies ist nicht der Fall. Eine einfache Heuristik zur Unterscheidung liefert die Wahl des Interrogativpronomens. Im Falle der temporalen Lesart rahmensetzender Modifikatoren ist das temporale Interrogativum Wann die beste Wahl, während für situationsexterne Modifikatoren ausschließlich das lokale Interrogativpronomen Wo in Frage kommt. Betrachten wir z.B. die temporale Interpretation des Rahmenadverbials in (17.a), die besagt: Als er im Bett war, sortierte John die Schmerzen des Tages. Die Fragevariante (17.b) ist in diesem Fall nicht nur zulässig, sondern gegenüber (17.c) deutlich präferiert.

(17) a. Im Bett sortierte John die Schmerzen des Tages. (Sten Nadolny: Die Entdeckung der Langsamkeit)

b. Wann sortierte John die Schmerzen des Tages?

c. ?Wo sortierte John die Schmerzen des Tages?

Ganz anders im Fall eines situationsexternen Modifikators wie in (18.a): Hier ist ausschließlich die Fragevariante (18.c) möglich. Läßt man (18.b) als Frage nach dem Lokaladverbial in (18.a) zu, so nur unter der Maßgabe einer - prosodisch reflektierten - Reinterpretation des Lokaladverbials als rahmensetzenden Modifikator. In diesem Fall wird (18.a) als Aussage über die Aktivitäten des Pfarrers zu der Zeit, als er sich vor der Kirche befand, verstanden. ${ }^{10}$

(18) a. Der Pfarrer hat vor der Kirche ein Schaf gebrandmarkt.

b. *Wann hat der Pfarrer ein Schaf gebrandmarkt?

c. Wo hat der Pfarrer ein Schaf gebrandmarkt?

Mit dem Inferenzverhalten und der Wahl des Interrogativpronomens Wann haben wir ein einfaches diagnostisches Mittel, um rahmensetzende, d.h. propositionsbezogene, Modifikatoren von situationsbezogenen Modifikatoren zu unterscheiden. Wie stellt sich vor diesem Hintergrund die Kombination von Kopula-Prädikativ-Konstruktionen mit lokalen Modifikatoren dar? Zunächst einmal zeigt sich, daß die in (9) aufgeführten Belege zur Gruppe der rahmensetzenden Modifikatoren gehören.

(9) a. Heidi war im Auto müde/betrunken/hungrig.

rahmensetzender Modifikator

b. ??Heidi war im Auto Vegetarierin/intelligent/blond.

Im Falle der Stadienprädikate in (9.a) liefert die temporale Lesart des Modifikators die präferierte Interpretation. (9.a) macht damit eine Aussage über Heidi zu der Zeit als sie sich im Auto befand. Unter den geeigneten kontextuellen Rahmenbedingungen sind aber auch andere Ausbuchstabierungen des rahmensetzenden Modifikators möglich. In einem Kontext beispielsweise, in dem eine Gruppe von Personen sich während einer Autofahrt Geschichten über Heidi ausdenkt oder nach Erklärungen für Heidis Verhalten sucht, bietet sich eine epistemische Interpretation des rahmensetzenden Modifikators an. Diese Option besteht ebenso für die Individuenprädikate in (9.b). Die Bewertung für (9.b)

${ }^{10}$ Eine weitere Rettungsmöglichkeit für das Paar (18.a/b) kann darin bestehen, die PP vor der Kirche als Temporaladverbial im Sinne von "vor dem Gottesdienst" $\mathrm{zu}$ interpretieren. In diesem Fall bezeichnet die DP nicht das Kirchgebäude, sondern die darin stattfindenden Prozesse; s. Bierwisch (1982, 1983). Für unsere Fragestellung ist allein die Interpretation der PP als Lokaladverbial einschlägig. 
kann also nicht der Grammatikalität dieser Sätze gelten, sondern sie bezieht sich lediglich auf die reduzierte Akzeptabilität des rahmensetzenden Modifikators bei temporaler Interpretation. In (9'.b) hingegen ist diese Interpretation weit weniger problematisch. ${ }^{11}$

(9') b. Heidi war in Portugal Vegetarierin/intelligent/blond.

Vom grammatischen Standpunkt aus können wir damit festhalten, daß SLP und ILP gleichermaßen durch rahmensetzende Lokaladverbiale modifizierbar sind. Akzeptabilitätsschwankungen beziehen sich lediglich auf die Verträglichkeit mit einer bestimmten Spezifizierungsoption rahmensetzender Modifikatoren, nämlich der temporalen Lesart. In Abschnitt 3.2 werde ich eine pragmatische Erklärung für die Kontraste in (9) geben.

Aus der Kombination von Kopula-Prädikativ-Konstruktionen mit rahmensetzenden, also propositionsbezogenen, Modifikatoren ergeben sich noch keine Hinweise für die An- bzw. Abwesenheit eines Situationsarguments. Aufschluß hierüber kann erst die Kombinierbarkeit mit situationsexternen Modifikatoren geben. Und hier zeigt sich, daß weder SLP noch ILP diese Modifikatoren zulassen; s. (19) und (20). (Das Temporaladverbial gerade bzw. die temporalen Nebensätze verhindern ein Ausweichen auf die Interpretation des Lokaladverbials als rahmensetzenden Modifikator.)

(19) a. *Heidi ist (gerade) in der Küche ohnmächtig.

SLP \& situationsexterner Modifikator

b. *Heidi ist (gerade) in der Hängematte wach.

c. *Heidi war im Auto betrunken, als sie von der Polizei angehalten wurde.

d. *Heidi ist (gerade) dort drüben hungrig.

e. *Heidi ist (gerade) auf dem Sofa müde.

(20) a. *Heidi ist (gerade) vor dem Spiegel eitel/blond.

ILP \& situationsexterner Modifikator

b. *Heidi war am Schreibtisch intelligent, als plötzlich das Telefon klingelte.

Nach den Ausführungen in Abschnitt 1 sollten zumindest die Stadienprädikate in (19) mit einem situationsbezogenen Modifikator kombinierbar sein. Diese Erwartung erfüllt sich nicht. KopulaPrädikativ-Konstruktionen unterscheiden sich damit klar von regulären Zustandsverben, die derartige Modifikatoren ohne weiteres zulassen; s. (21).

(21) a. Heidi schläft (gerade) in der Hängematte.

b. Heidi wartet (gerade) im Auto.

c. Heidi ruht (gerade) auf dem Sofa.

d. Der Lieferwagen parkt (gerade) in der Einfahrt.

e. Das Boot ankerte (gerade) in der Bucht.

Gegen die Beurteilung der Sätze (19) und (20) mag man einwenden, daß die Varianten in (22) durchaus grammatisch sind. Tatsächlich liegen hier jedoch andere Strukturen zugrunde. In (19) und (20) dient das Adjektiv als Prädikativ, und das Lokaladverbial ist situationsexterner Modifikator. Bei (22) hingegen nimmt das Lokaladverbial die Prädikativposition ein, und das Adjektiv bildet ein (depiktives) sekundäres Prädikat. Depiktive Prädikate zeichnen sich gerade dadurch aus, daß sie nicht den verbalen Referenten weiter beschränken, sondern eines der Argumente des Verbs, hier: das Subjekt.

\footnotetext{
11 Eine Analyse à la Diesing $(1992,1992 a)$ wird diesen Beobachtungen in zweifacher Hinsicht nicht gerecht. Erstens behandelt sie einen rahmensetzenden Modifikator in Kombination mit einem SLP wie einen situationsexternen Modifikator und ignoriert damit die Unterschiede zwischen der pragmatisch gesteuerten temporalen Interpretation rahmensetzender Modifikatoren und der grammatisch festgelegten Bedeutung situationsexterner Modifikatoren. Zweitens reduziert sie die Interpretation rahmensetzender Modifikatoren in Kombination mit ILP auf die syntaktisch vorgegebene Beschränkung einer bestimmten Quantorendomäne (s. (10)) und kann damit nicht die ganze Bandbreite zulässiger Interpretationen rahmensetzender Modifikatoren erfassen. Kurz: Diesing-Ansätze verkennen die Rolle der Pragmatik bei der Ermittlung der Äußerungsbedeutung rahmensetzender Modifikatoren und gelangen deshalb zu falschen Einschätzungen des grammatischen Bedeutungsanteils.
} 
(22) a. Heidi ist ohnmächtig in der Küche.

b. Heidi war betrunken im Büro.

Die Inkompatibilität von Kopula-Prädikativ-Konstruktionen mit situationsbezogenen Modifikatoren betrifft nicht nur Lokaladverbiale, sondern setzt sich fort bei Komitativen, Manner-Angaben und situationsbezogenen Partizipien (s. Zimmermann 1999); s. (23) vs. (24).

(23) a. *Heidi war mit ihrer Nichte hungrig.

b. *Heidi war mit großer Geduld nüchtern.

c. *Heidi war ausgestreckt auf dem Sofa müde.

(24)

Heidi wartete $\left.\begin{array}{ll}\{\text { mit ihrer Nichte } \\ \text { mit großer Geduld } \\ \text { ausgestreckt auf dem Sofa }\end{array}\right\}$ auf den Anruf.

Zur Unverträglichkeit mit Manner-Angaben gehört auch, daß Kopula-Prädikativ-Konstruktionen nicht in Wie-Fragen (im Sinne von: "In welcher Art und Weise?") auftreten; s. (25) vs. (26). ${ }^{12}$

(25) *Wie war Heidi hungrig/faul/müde?

(26) Wie hat Heidi gewartet/geruht/geschlafen?

Unter der Annahme eines Situationsarguments für Kopula-Prädikativ-Konstruktionen sind die Kontraste (19)/(20) vs. (21) sowie (23) vs. (24) und (25) vs. (26) völlig unerwartet. Von den Modifikatoren, die sich dem VP/IP-Bereich zuordnen lassen, mit Ausnahme also von Satzmodifikatoren, sind einzig temporale Modifikatoren in der Umgebung von Kopula-Prädikativ-Konstruktionen zugelassen. Hier treffen wir auf ähnliche Akzeptabilitätsunterschiede wie bei der oben diskutierten temporalen Interpretation rahmensetzender Modifikatoren s. (27) - (29).

\begin{tabular}{|c|c|c|}
\hline & gestern & ) \\
\hline \multirow[t]{4}{*}{ Heidi war } & $\{$ drei Stunden lang & Imüde/betrunken/hungrig. \\
\hline & nach einem Glas Wein & | \\
\hline & selten & j \\
\hline & gestern & ) \\
\hline \multirow[t]{4}{*}{ ??Heidi war } & $\{$ drei Stunden lang & Vegetarierin/intelligent/blond. \\
\hline & nach diesem Vorfall & l \\
\hline & I selten & J \\
\hline & I seit frühster Kindheit & ) \\
\hline Heidi war & $\left\{\begin{array}{l}\text { während ihres Studiums } \\
\text { nie }\end{array}\right.$ & Vegetarierin/intelligent/blond. \\
\hline
\end{tabular}

Auch in diesem Fall halte ich eine pragmatische Erklärung derartiger Akzeptabilitätsschwankungen für angezeigt; s. Abschnitt 3.2.

Die Eigenschaft, keine situationsbezogenen Modifikatoren zuzulassen, teilen Kopula-PrädikativKonstruktionen mit den sogenannten statives, Verben, die im SLP/ILP-Paradigma als Individuen-

12 Im Falle von (i) erkundet die Frage nicht die Art und Weise, in welcher die betreffende Eigenschaft sich manifestiert, sondern den Ausprägungsgrad der Eigenschaft. Mögliche Antworten auf (i) beinhalten Spezifizierungen der prädikativen AP wie in (ii). Antworten für (26) hingegen sind durch adverbiale Modifikatoren der VP gekennzeichnet; s. (iii). (S. Bayer (1986) zu weiteren möglichen Einwänden gegen (25).)

(i) Wie hungrig/faul/müde war Heidi?

(ii) Heidi war sehr/ziemlich/nur ein bißchen/ausgesprochen/über alle Maßen müde.

(iii) Heidi hat gut/ausgezeichnet/fest/tief/unruhig/traumlos geschlafen. 
prädikate ausgewiesen sind; s. (6). Auf die Gemeinsamkeiten dieser Verben mit Kopula-PrädikativKonstruktionen werde ich genauer in Abschnitt 2.3. eingehen. Hier seien vorab die relevanten Daten aufgeführt. So lassen stative Verben weder situationsexterne lokale Modifikatoren zu (s. (30)) $)^{13}$ noch sind sie mit Komitativen, Manner-Angaben oder situationsbezogenen Partizipien kombinierbar; s. (31).

(30) a. *Heidi ähnelt (gerade) an der Straßenecke ihrer Nichte.

b. *Vor seiner entsetzten Frau heißt dieser Mann Hermann Saumweber.

c. *Eine Flasche italienischer Rotwein kostet neben dem französischen Rotwein 15 DM.

(31) a. *Heidi ähnelt mit ihrer Nichte Romy Schneider.

b. *Vor ihrer Heirat hieß Heidi ohne große Anstrengung/mit Inbrunst Regenbogen.

c. *Eine Flasche italienischer Rotwein kostet (ja doch) sorgfältig ettikettiert 15 DM.

Die Kombination von Kopula-Prädikativ-Konstruktionen mit lokalen und vergleichbaren anderen Modifikatoren stellt sich nach den obigen Ausführungen in einem neuen Licht dar. Für die im Stadien/Individuen-Paradigma behaupteten Unterschiede zwischen SLP und ILP hinsichtlich der Zulässigkeit lokaler Modifikatoren finden sich im Bereich der Kopula-Prädikativ-Konstruktionen keine Belege. Vielmehr ergibt sich ein einheitliches Bild, wonach rahmensetzende, d.h. propositionsbezogene, Modifikatoren grammatisch grundsätzlich zugelassen, situationsbezogene Modifikatoren hingegen grundsätzlich ausgeschlossen sind.

(32) Kombination von Kopula-Prädikativ-Konstruktionen mit lokalen Modifikatoren:

- grammatisch: SLP/ILP \& rahmensetzender Modifikator

- ungrammatisch: SLP/LLP \& situationsexterner Modifikator

Die in 1.1 wiedergegebene Einschätzung Chierchias (1995), wonach temporäre, nicht aber permanente Eigenschaften im Raum lokalisiert seien, wird durch das Sprachsystem jedenfalls nicht gestützt. Temporäre und permanente Eigenschaften von Individuen erweisen sich als gleichermaßen ortsungebunden. Hinsichtlich der Kombination mit situationsbezogenen Modifikatoren macht das Sprachsystem allerdings einen klaren Unterschied zwischen temporären oder permanenten Eigenschaften von Individuen einerseits und Zuständen wie in (21), (24) und (26) andererseits. Diesem Unterschied werden die in Abschnitt 1 geschilderten Ansätze nicht gerecht.

Ich werte die Inkompatibilität von ILP und SLP mit lokalen und anderen situationsbezogenen Modifikatoren als einen Hinweis darauf, daß Kopula-Prädikativ-Konstruktionen nicht mit einem Situationsargument ausgestattet sind. Die hier aufgezeigte Unverträglichkeit sollte umso ernster genommen werden, als die Kombination mit Modifikatoren seit Davidson zu den zentralen Motiven für die Einführung von Situationen als raumzeitlichen Entitäten zählen. Bevor wir die Konsequenzen dieses Befundes für die Semantik von Kopula-Prädikativ-Konstruktionen ausloten, will ich weitere Argumente vorbringen, die diesen ersten Befund stützen.

\subsection{Wahrnehmungsverben}

Einen zweiten Testfall für die Frage des Situationsbezugs von Kopula-Prädikativ-Konstruktionen stellen Wahrnehmungsverben dar. Gemäß Higginbotham (1983) bezeichnen Infinitivkomplemente von Wahrnehmungsverben Situationen; s. auch Bayer (1986). Als Entitäten "in der Welt" können Situationen Gegenstand direkter Wahrnehmung sein. Die Sätze in (33) drücken also die unmittelbare

13 Rahmensetzende Modifikatoren sind hingegen, wie auch im Fall der Kopula-Prädikativ-Konstruktionen zulässig; s.

(i) - (iii).

(i) Bei Kerzenlicht ähnelt Heidi ihrer Nichte.

(ii) In der Wiener Staatsoper heißt der Souffleur "Maestro Suggeritore".

(iii) Eine Flasche italienischer Rotwein kostet im Restaurant 35 DM. 
Wahrnehmung des durch das jeweilige Infinitivkomplement bezeichneten Zustandes bzw. Prozesses aus. ${ }^{14}$

(33) a. Heidi sah Luise nachdenken.

b. Heidi sah Luise sich langweilen.

c. Heidi sah das Buch auf dem Tisch liegen.

d. Heidi hörte Luise flüstern.

e. Heidi hörte Luise atmen.

Wenn Kopula-Prädikativ-Konstruktionen tatsächlich mit einem Situationsargument ausgestattet wären, so sollten sie als Infinitivkomplemente von Wahrnehmungsverben auftreten können. Dies ist wiederum nicht der Fall; s. (34).

(34) a. *Heidi sah Luise blond sein.

b. *Heidi sah Luise gelangweilt sein.

c. *Heidi sah das Buch auf dem Tisch sein.

d. *Heidi hörte die Callas heiser sein.

e. *Heidi hörte das Klavier verstimmt sein.

Die Konstellationen in (34) wurden dabei so gewählt, daß ihrer Wahrnehmbarkeit nichts im Wege stehen sollte. Demgegenüber sind die Voraussetzungen speziell bei (33.a/b) deutlich schlechter. Um so bemerkenswerter ist der Grammatikalitätskontrast in (33) und (34).

Bayer (1986: 3) vermutet, daß die Ungrammatikalität von Ausdrücken des Typs (34) auf das Fehlen kognitiv relevanter Grenzen bei dem in Frage stehenden Gegenstand der Wahrnehmung zurückzuführen ist. Hierin sieht er den Grund dafür, daß das Infinitivkomplement keine Situation bezeichnet. Dazu ist jedoch anzumerken, daß selbst bei expliziter Setzung temporaler Grenzen die KopulaPrädikativ-Konstruktionen ungrammatisch bleiben; s. (35).

(35) a. *Heidi sah Luise zehn Minuten lang gelangweilt sein.

b. *Heidi sah Luise um 3 Uhr im Café sein.

c. *Heidi hörte die Callas während der Rachearie heiser sein.

Kopula-Infinitive führen offensichtlich keinen geeigneten Wahrnehmungsgegenstand ein und erfüllen somit nicht die Voraussetzungen, um als Komplemente von Wahrnehmungsverben auftreten zu können. Der Kontrast in (36) unterstreicht diesen Befund. Unsere sprachlichen Mittel erlauben nur die Wiedergabe des Gesehenen als Objektwahrnehmung mit dem Adjektiv als sekundärem Prädikat wie in (36.a). Die für Situationswahrnehmung reservierte Form in (36.b) ist ausgeschlossen.

(36) a. Heidi sah Luise nackt/nachdenklich.

b. *Heidi sah Luise nackt/nachdenklich sein.

Im Hinblick auf die Kombination mit Wahrnehmungsverben zeigt sich erneut, daß die gesamte Gruppe der Kopula-Prädikativ-Konstruktionen sich einheitlich verhält. Und wiederum stimmt dieses

\footnotetext{
14 Bayer (1986) unterscheidet zwischen Situationswahrnehmung (bzw. in seiner Redeweise "Ereigniswahrnehmung"), wie sie bei den hier diskutierten Wahrnehmungsverben mit Infinitivkomplementen vorliegt (s. (i)), und "epistemisch befrachteter" Faktenwahrnehmung. Letztere wird durch Wahrnehmungsverben mit daß-Komplementen ausgedrückt; s. (ii). Hier sind natürlich auch Kopula-Konstruktionen und stative Verben möglich; s. (iii)/(iv). Situationswahrnehmung setzt voraus, daß die Wahrnehmungszeit in die Situationszeit fällt, für Faktenwahrnehmung gilt diese Beschränkung nicht; vgl. (v)/(vi). Zu weiteren Differenzen s. Bayer (1986: 11ff). Für unsere Fragestellung ist nur die durch Infinitivkomplemente angezeigte Situationswahrnehmung relevant.

(i) Heidi sieht Luise Mambo tanzen.

(ii) Heidi sieht, daß Luise Mambo tanzt.

(iii) Heidi sieht, daß die Aprikosen teuer sind.

(iv) Heidi sieht, daß Luise ihrer Nichte ähnelt.

(v) Heidi sieht Luise Mambo *tanzen werden / *getanzt haben.

(vi) Heidi sieht, daß Luise Mambo tanzen wird / getanzt hat.
} 
Verhalten mit demjenigen stativer Verben überein: Auch stative Verben treten nicht als Komplemente von Wahrnehmungsverben auf; s. (37).

(37) a. *Luise sah/hörte Heidi ganz bcmerkenswert Romy Schneider ähneln.

b. *Luise sah/hörte Robin Hood "der Rächer der Enterbten" heißen.

c. *Luise sah/hörte den Rotwein 35 DM kosten.

d. *Luise sah/hörte Heidi des Rätsels Lösung wissen.

Das Verhalten von Kopula-Prädikativ-Konstruktionen im Kontext von Wahrnehmungsverben bestätigt damit die Erkenntnisse zur Kombination mit Modifikatoren und nährt die Zweifel am Situationsbezug von Kopula-Prädikativ-Konstruktionen.

\subsection{Kopula-Prädikativ-Konstruktionen vs. Zustandsausdrücke}

Ein wesentliches Anliegen des vorliegenden Beitrages ist es zu zeigen, (a) daß Kopula-PrädikativKonstruktionen sich grammatisch einheitlich verhalten, unabhängig davon, ob sie temporäre oder permanente Eigenschaften bezeichnen, und (b) daß sich ihr grammatisches Verhalten von dem regulärer Zustandsausdrücke unterscheidet. Es ist daher an der Zeit, mein Verständnis von Zustandsausdrücken zu präzisieren. Es geht mir dabei um einen Unschärfebereich (bzw. eine Lücke) in der Vendlerschen Verbklassifikation. Dieser betrifft die unter (38) aufgeführten Verben.

(38) Reguläre Zustandsverben:

Positionsverben: sitzen, stehen, liegen, hocken, hängen, lehnen, ...

schlafen, wachen, ruhen, leben, warten, lauern, kleben, ankern, parken, ...

Bei Vendler (1967) selbst finden derartige Verben keine Erwähnung. Der Versuch einer Einordnung führt zu widersprüchlichen Resultaten. Das Kriterium der Progressivierbarkeit (ihrer englischen Pendants) unterscheidet sie von state terms, zu denen Vendler die Verben in (39) rechnet. Dieses sind die in den vorigen Abschnitten erwähnten statives. (Die monierte Unschärfe spiegelt sich auch in der Handhabung der Termini "state term" und "stative" teils als Synonyme teils als Gegensatzpaar im Bereich statischer Ausdrücke wider.)

(39) Statives (Vendlers state terms):

wissen, glauben, lieben, hassen, sein \& Prädikativ, ...

Damit käme für die Verben vom Typ (38) eine Zuordnung zur Klasse der activitiy terms bzw. allgemeiner: der Prozeßverben (s. Mourelatos 1978) in Frage; s. (40).

(40) Prozeßverben (Vendlers activity terms):

Bewegungsverben: laufen, gehen, fahren, ...

Handlungsverben: essen, lesen, schreiben, ...

Witterungsverben: regnen, schneien, winden, ...

...

Diese Einteilung kollidiert jedoch mit der seit Vendler (1967) üblichen Feststellung, daß states zu atomaren Zeiten, sprich: Zeitpunkten, bestehen, activities hingegen nur zu nicht-atomaren Zeiten, sprich: Zeitintervallen; s. z.B. Dowty (1979), Herweg (1990), Egg (1994). Hinsichtlich ihrer Teilintervalleigenschaften verhalten sich die Verben in (38) wie state terms. ${ }^{15}$ In der Folge hat

15 Dowty (1979) und im Anschluß daran Egg (1994) erwägen, Verben vom Typ (38) als intervallbasiert aufzufassen. Sie argumentieren damit, daß man ein Zeitintervall heranziehen muß, um zweifelsfrei feststellen zu können, ob z.B. ein Ball auf einem Teppich liegt oder nicht vielmehr über den Teppich rollt. Ich halte diese Argumentation nicht für überzeugend. Erstens gilt dies für die klassischen state-Fälle in (39) ebenso. Um uns zu vergewissern, ob ein Licht tatsächlich rot ist und nicht nur flackert oder ob ein Augenblick der Liebe nicht vielmehr Teil einer Haßliebe ist, sind wir gut beraten, auch hier größere Zeitintervalle für die Beurteilung zugrundezulegen. Zweitens scheinen mir die Bedingungen, unter denen wir in der Lage sind, eine bestimmte Eigenschaft zu erkennen, hier nicht von primärem Interesse. Ich halte vielmehr die Schlußfolgerungen für maßgeblich, die sich aus dem Zutreffen der in Frage stehenden 
beispielsweise Dowty (1979) Vendlers state-Kategorie u.a. um Verben vom Typ (38) erweitert; s. die Aufstellung in Dowty (1979: 66f). Ebenso verfährt Bach (1986), indem er innerhalb der Kategorie der Zustände zwischen dynamischen Zuständen, für die er als Beispiele Positionsverben aufführt, und statischen Zuständen, den klassischen statives, unterscheidet. Beide Zustandstypen machen zusammen mit Prozessen und Ereignissen Bachs Eventualitäten aus. In späteren Arbeiten sind die bei Dowty (1979: 180ff) und Bach (1986: 6) noch durch Subtypen kenntlich gemachten Unterschiede innerhalb dieser neuen state-Kategorie dann zunehmend aus dem Blickfeld geraten (s. z.B. Herweg (1990), Kamp \& Reyle (1993) sowie die in Abschnitt 1.1 genannten Ansätze) oder durch neue Einteilungen entlang der Stadien/Individuen-Demarkation überschrieben worden; s. (5)/(6).

Ich halte die Zusammenfassung von Zustandsverben vom Typ (38) und statives vom Typ (39) zu einer Kategorie für eine Mesalliance. Dies belegen bereits die in den beiden vorangehenden $\mathrm{Ab}$ schnitten aufgezeigten Unterschiede hinsichtlich der Kombinierbarkeit mit situationsbezogenen Modifikatoren sowie dem Auftreten als Komplement von Wahrnehmungsverben. Ebensowenig kann die Vereinnahmung der Zustandsverben unter die Kategorie der Prozeßverben ernsthaft ins Auge gefaßt werden. Hierzu seien - neben den unterschiedlichen Teilintervalleigenschaften - exemplarisch zwei sprachliche Kontexte genannt, die Prozeßausdrücke, nicht aber Zustandsausdrücke zulassen: anaphorischer Aufgriff mit geschehen/passieren s. (41) vs. (42) und Modifikation durch die MannerAdverbien schnell/langsam s. (43) vs. (44).

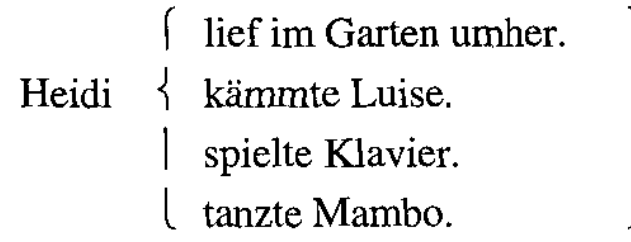

Das geschah/passierte während ...

Heidi stand am Fenster.

Heidi wartete auf Luise.

Heidi lebte in La Paz.

Heidi schlief.

Die Wäsche hing auf der Leine.

*Das geschah/passierte während ... ${ }^{16}$

Das Buch lag auf dem Tisch.

( Das Boot ankerte in der Bucht. )

(43) a. Heidi lief schnell/langsam im Garten umher.

b. Heidi schmückte den Weihnachtsbaum sehr schnell/langsam.

Verben zu einem gegebenen Zeitintervall ergeben. Im Falle der Zustandsausdrücke vom Typ (38) und Vendlers state terms gilt die Teilintervalleigenschaft unbeschränkt, bei Prozeßverben muß sie jedoch auf ein bestimmtes Intervallminimum begrenzt werden.

${ }^{16}$ Die Angabe der genauen Selektionsbeschränkungen von geschehen und passieren bedarf weiterer Klärung. So können beide Verben (insbesondere in Kombination mit Frequenzadverbien) als Synonyme zu vorkommen verwendet werden. In diesem Fall ist die Kombination mit Zustandsverben (s. (i)/(ii)) und auch statives (s. (iii) - (v)) zulässig.

(i) weil es häufig geschieht/passiert/vorkommt, daß Heidi in der Dämmerung am Fenster steht

(ii) weil es immer wieder geschah/passierte/vorkam, daß auf dem Nachttisch Aprikosenkerne lagen

(iii) weil es sogar geschieht/passiert/vorkommt, daß was Eßbares im Kühlschrank ist

(iv) weil es immer wieder geschieht/passiert/vorkommt, daß jemand Valentin heißt

(v) Heidi war sprachlos. Das geschah/passierte/kam ganz selten vor.

Im Falle von (41)/(42) dienen die Proverben geschehen/passieren zum Aufgriff eines spezifischen Situationsreferenten; vorkommen ist in diesem Kontext ausgeschlossen. Ich will hier auf die Verwendungsvariante in (i) - (v) nicht weiter eingehen. Der Grammatikalitätsunterschied zwischen (41) und (42) scheint mir hinreichend ausgeprägt, um ihn als ein Indiz dafür zu werten, daß die Unterscheidung Zustand vs. Prozeß sprachlich reflektiert wird. 
(44) a. *Die Briefmarke klebte schnell/langsam auf dem Brief. ${ }^{17}$

b. ${ }^{*}$ Heidi wartete sehr schnell/langsam auf Luise.

Die Resultate der bisher angewandten Tests erlauben die folgende Schlußfolgerung: Zustände teilen mit Prozessen und Ereignissen (Vendlers accomplishments und achievements) u.a. die Eigenschaften, räumlich lokalisiert und Gegenstand der Wahrnehmung zu sein. Die entsprechenden sprachlichen Ausdruicke lassen (situationsbezogene) lokale Modifikatoren sowie Manner-Angaben zu und treten als Infinitivkomplemente von Wahrnehmungsverben auf. Es ist mithin plausibel, Zustände, Prozesse und Ereignisse als Ausprägungen von Situationen aufzufassen. Für Kopula-Prädikativ-Konstruktionen als Vertretern der statives können wir zunächst konstatieren, daß sie die in Frage stehenden Eigenschaften nicht aufweisen. In diesem Zusammenhang seien als weitere Unterschiede zwischen Zustandsverben und statives das Verhalten bei Nominalisierung und in der tun-Periphrase genannt. Obschon nicht dafür prädestiniert (da nicht agentiv), lassen Zustandsverben (gleich Prozeß- und Ereignisverben) die Bildung von Nominalisierungen auf der Basis der Derivationsaffixe -erei und Ge$e \mathrm{zu}$; s. (45). (Diese Nominalisierungstypen gelten als Indiz für den agentiven Charakter der zugrundeliegenden Verben.) Für Kopula-Prädikativ-Konstruktionen und stative Verben trifft dies nicht zu; s. (46).
( Diese Warterei
| Diese Bis-in-die-Puppen-Schlaferei
\{ Diese Am-Kamin-Hockerei \}
Dieses Rumgesitze
( Dieses Rumgehänge auf Partys
$\left\{\begin{array}{l}\text { Diese *Albern-Seierei } \\ \text { Diese *Enttäuscht-Seierei }\end{array}\right.$
a. $\{$ Diese *Im-Garten-Seierei $\}$ nervt Heidi.
Dieses *Im-Büro-Geseie
Dieses *Schlecht-gelaunt-Geseie
( Diese *An-Sternzeichen-Glauberei \}
Diese *Pasta-Mögerei
b. $\{$ Diese $*$ Zuviel-Wiegerei
nervt Heidi.
Dieses *Romy-Schneider-Geähnele |
Dieses *In-den-Himmel-Gerage
Dieses *9,99DM-Gekoste

Ebenso lassen jene Dialekte des Deutschen, in denen die tun-Periphrase eine aspektuelle Funktion hat, Zustandsverben in dieser Konstruktion marginal zu, Kopula-Prädikativ-Konstruktionen und stative Verben bleiben jedoch ausgeschlossen; s. (47) vs. (48).18

\footnotetext{
17 Im Falle von schnell besteht bei (44) die Möglichkeit einer ingressiven Uminterpretation des Verbs; s. z.B. Herweg (1990). In diesem Fall charakterisiert das Adjektiv einen Prozeß, der zum Eintreten des bezeichneten Zustandes führt. $\mathrm{Daß}$ es sich hierbei um eine nicht-reguläre Bedeutungskonstitution handelt, wird auch daran ersichtlich, daß diese Option für langsam offenbar nicht besteht.

18 Bei Topikalisierung des VP-Komplements wie in (i)/(ii) büßt tun seine aspektuelle Funktion ein und dient nurmehr zur Modusmarkierung; vgl. (iii).

(i) Italienische Opern kennen tut Heidi viele, aber mitsingen würde sie trotzdem nie.

(ii) Auf Luise böse sein tut Heidi nicht gerade, aber gekränkt ist sie schon.

(iii) Ich tät an Deiner Stelle auf Luise böse sein.
} 
(47) a. 'Heidi tut verlegen in der Ecke stehen.

b. 'Heidi tut ganz brav auf der Bank sitzen.

c. ?Heidi tut geduldig auf Luise warten.

d. ?Das Buch tut schon die ganze Zeit auf dem Tisch liegen.

(48) a. *Heidi tut auf Luise böse sein.

b. *Heidi tut schrecklich durstig sein.

c. *Heidi tut viele italienische Opern kennen.

d. *Das Buch tut schon die ganze Zeit auf dem Tisch sein.

Die Divergenzen zwischen Kopula-Prädikativ-Konstruktionen und Zustandsausdrücken vom Typ (38) scheinen mir damit hinreichend belegt. Im gleichen Zuge wurden die Argumente dafür, daß das Sprachsystem die Unterscheidung temporärer vs. permanenter Eigenschaften von Individuen registriert, entkräftet. Kopula-Prädikativ-Konstruktionen gehören einheitlich zur Klasse der statives. Dieser Beobachtung werden die in Abschnitt 1 genannten Ansätze nicht gerecht.

\section{Eine alternative Analyse}

Es bedarf also eines Alternativ-Vorschlags für die Interpretation von Kopula-Prädikativ-Konstruktionen. Im folgenden sollen die dabei zu prüfenden Möglichkeiten vorgestellt und ein vorläufiger Vorschlag unterbreitet werden. Dies beinhaltet zum einen den Entwurf einer Semantik für KopulaPrädikativ-Konstruktionen, die ihren Charakter als statives erfaßt, und zum anderen eine pragmatische Erklärung für die bei Stadien- und Individuenprädikaten - bleiben wir der Bequemlichkeit halber bei dieser grammatisch allerdings folgenlosen Benennung - in Kombination mit rahmensetzenden Modifikatoren zuweilen auftretenden Akzeptabilitätsschwankungen.

\subsection{Kopula-Prädikativ-Konstruktionen als Statives}

Wie lassen sich die in Abschnitt 2 aufgezeigten grammatischen Unterschiede zwischen KopulaPrädikativ-Ausdrücken und Zustandsausdrücken angemessen rekonstruieren? Wenn wir die Charakterisierung von Situationen als raumzeitliche Entitäten ernst nehmen, müssen wir für Situationsausdrïcke fordern, daß sie lokale Modifikatoren zulassen und als Komplemente von Wahrnehmungsverben auftreten. Die Konsequenz kann dann nur sein, daß Kopula-Prädikativ-Ausdrücke, ob sie nun temporäre oder permanente Eigenschaften ausdrücken, eben keine Situationen bezeichnen. Die Zulässigkeit von temporalen Modifikatoren (s. (27) - (29)), ebenso wie die Präsenz von Tempus, weisen allerdings darauf hin, daß Kopula-Prädikativ-Ausdrücke Zeitbezug haben. Eine Semantische Form der Kopula sein, die diesem Umstand Rechnung trägt, ist in (49) angegeben.

(49) sein: $\lambda \mathrm{P} \lambda \mathrm{x} \lambda \mathrm{t}[\mathrm{P}(\mathrm{x}) \mathrm{AT} \mathrm{t}]$

(mit $\mathrm{t}$ als Variable über Zeiten)

Die SF in (49) sieht statt des Situationsarguments in (1) ein Zeitargument vor. (49) besagt, daß die durch das Prädikativ gegebene Eigenschaft $P$ auf das Subjekt $x$ zur Zeit $t$ zutrifft. (AT ist eine Relation zwischen einer Proposition $\mathrm{p}$ und einem Zeitintervall $\mathrm{t}$, die wahr ist gdw. $\mathrm{p}$ wahr ist zu $\mathrm{t}$.) Als SF für die VP des Satzes in (50.a) ergibt sich unter Zugrundelegung von (49) die Struktur in (50.b).

(50) a. Heidi ist müde.

b. VP: $\lambda t$ [TIRED (Heidi) AT t]

Das heißt, im Falle einer Kopula-Prädikativ-Konstruktion denotiert die VP eine Menge von Zeiten, zu denen die betreffende Eigenschaft vorliegt. Die Präsenz des Zeitarguments schafft die Voraussetzungen für die Integration von Tempus und ggf. temporalen Modifikatoren. Die Unterschiede zwischen Kopula-Prädikativ-Ausdrücken und Situationsausdrücken sind damit auf den Bereich der 
VP eingegrenzt. Die anschließenden funktionalen Etagen sind davon nicht betroffen; s. den Vorschlag zur kompositionalen Semantik der funktionalen Projektionen des Verbs in Maienborn (1998). Diese Analyse prognostiziert damit, daß das Verhalten von Kopula-Prädikativ-Konstruktionen im Hinblick auf Modifikatoren, die dem CP/IP-Bereich zugeordnet sind, sich nicht von demjenigen der Zustandsverben unterscheidet. Dies gilt z. B. für die rahmensetzenden Modifikatoren in Abschnitt 2.1, die gleichermaßen bei stativen wie bei situativen Ausdrücken auftreten. Die SF in (49) bietet hingegen keine Anschlußmöglichkeiten für lokale oder andere situationsbezogene Modifikatoren ${ }^{19}$ und erfüllt nicht die Argumentanforderungen von Wahrnehmungsverben. ${ }^{20}$ (49) kann also als Basis für das in Abschnitt 2 diagnostizierte Verhalten von Kopula-Prädikativ-Konstruktionen dienen.

In Analogie zu (49) können wir als SF für ein statives Vollverb wie ähneln die SF in (51) annehmen. Das heißt, ähneln bezeichnet eine Relation zwischen zwei Individuen, die zu einer Zeit $t$ vorliegt.

\section{(51) ähneln: $\quad \lambda \mathrm{y} \lambda \mathrm{x} \lambda \mathrm{t}[\operatorname{RESEMBLE}(\mathrm{x}, \mathrm{y}) \mathrm{AT} \mathrm{t}]$}

Der hier skizzierte Vorschlag führt das unterschiedliche grammatische Verhalten von Kopula-Prädikativ-Konstruktionen und Zustandsverben auf einen ontologischen Unterschied zurück. KopulaPrädikativ-Konstruktionen als Vertreter der statives bezeichnen zeitlich situierte Eigenschaften bzw. Relationen von Individuen. Zustandsverben hingegen bezeichnen, gleich Prozeß- und Ereignisverben, Situationen.

Dies ist ein in mehrerer Hinsicht legitimierungsbedürftiger Vorschlag. Zu klären ist insbesondere, ob sich aus bestimmten grammatischen Verhaltensweisen von Kopula-Prädikativ-Konstruktionen etwa im Bereich der Infinitivnominalisierungen oder der Kombination mit Kausaladverbialen und bestimmten Manner-Angaben (s. Jäger 1999) stichhaltige Argumente für eine Reifikation der durch das Vorliegen von Eigenschaften bzw. Relationen qualifizierten Zeitphasen ergeben. Ich werde im Rahmen dieses Beitrags nicht auf diese Punkte eingehen können, möchte aber kurz auf eine durch die oben erwähnten Klassifikationen von Dowty (1979) und Bach (1986) nahegelegte Umsetzungsvariante dieser Reifikation eingehen, die ich für problematisch, da kaum vereinbar mit den Grundannahmen des Davidsonschen Paradigmas, halte. Dies wäre eine Reifikation unter dem Dach der Situationen, derzufolge Kopula-Prädikativ-Konstruktionen einen Subtyp von Zuständen bezeichnen, Zustände eben, die nicht wahrnehmbar sind, nicht im Raum lokalisiert und deren Art und Weise nicht näher bestimmbar ist. Was bliebe in diesem Fall als kleinster gemeinsamer Nenner für die Charakterisierung von Situationen als ontologische Basiseinheiten? Nicht viel. Es ließe sich nur sagen, daß diese Entitäten eine zeitliche und eine irgendwie geartete inhaltliche Ausprägung haben. Kopula-Prädikativ-Ausdrücke gäben also den Ton an für die gesamte Klasse der Situationsausdrücke.

Zum Situationsbegriff im Davidsonschen Paradigma gehört ganz wesentlich, daß es sich hierbei um Entitäten "in der Welt" handelt, die über eine zeitliche sowie eine räumliche Dimension verfügen, und die eine kohärente Binnenstruktur aufweisen, in die etwaige Partizipanten funktional eingebunden sind. (Der letzte Punkt verdiente meines Erachtens bei der Diskussion zum Verständnis von Situationen mehr Aufmerksamkeit; s. hierzu die Überlegungen in Maienborn $(1996,1998)$ und Eckardt (1998)). Dieses Situationsverständnis hat sich seit Davidson bei aller Vorläufigkeit und

\footnotetext{
${ }^{19}$ Ich nehme die übliche Analyse intersektiver Modifikatoren an, derzufolge der Modifikator ein zusätzliches Prädikat über das referentielle Argument des Modifikanden liefert; s. z.B. (in Variationen) Higginbotham (1985), Bierwisch (1988), Zimmermann (1992, 1998), Jacobs (1995), Maienborn (1996, 1998), Heim \& Kratzer (1998). Die Integration situations bezogener Modifikatoren setzt demnach die Bereitstellung eines Situationsreferenten durch den Modifikanden voraus.

20 Die hier relevanten Aspekte der SF von sehen etwa ließen sich in dem in Abschnitt 1.1 eingeführten Rahmen wie folgt wiedergeben:

(i) sehen: $\lambda y \lambda x \lambda \mathrm{s}$ [s INST [x SEE y]] (mit y als Variable über Objekte oder Situationen) Damit liegt bei der Kombination mit einer Kopula-Prädikativ-Konstruktion ein semantischer Sortenkonflikt vor, der zur Ungrammatikalität führt.
} 
Lückenhaftigkeit als äußerst produktiv und hilfreich erwiesen. Es sollte nicht ohne zwingende Gründe aufgegeben werden. Mein Fazit lautet daher: Wie auch immer eine Reifikationslösung für KopulaPrädikativ-Konstruktionen (bzw. allgemeiner: statives) aussehen könnte, eine Subsumption unter die Kategorie der Situationen scheidet aus.

\subsection{Eine pragmatische Erklärung für den Stadien/Individuen-Kontrast bei Kopula- Prädikativ-Konstruktionen}

Wenden wir uns dem zweiten Problemkreis zu, den Akzeptabilitätsschwankungen bei KopulaPrädikativ-Konstruktionen in Kombination mit lokalen Modifikatoren, die im Stadien/IndividuenParadigma als Hinweis auf eine grammatische Unterscheidung temporärer vs. permanenter Eigenschaften gewertet werden. Die Überlegungen in Abschnitt 2.1 haben gezeigt, daß die anvisierten Unterschiede lediglich bei einer bestimmten Spezifizierungsvariante rahmensetzender Modifikatoren auftreten, nämlich der temporalen Interpretation. Sie sind damit nicht Gegenstand der Grammatik. Wie könnte nun eine pragmatische Erklärung der beobachteten Akzeptabilitätsschwankungen aussehen? Betrachten wir dazu zunächst (52).

(52) In Portugal war Luise reich.

Ich habe in Maienborn (1998) dafür argumentiert, als grammatisch vermittelten Bedeutungsbeitrag rahmensetzender Modifikatoren eine semantisch unterbestimmte Beschränkung der Anwendung des Kommentars auf das Topik anzusetzen. Die konkrete Ausbuchstabierung dieser Beschränkung erfolgt pragmatisch unter Hinzuziehung von Kontext- und Weltwissen. Im Falle der temporalen Lesart dient der rahmensetzende Modifikator zur näheren Bestimmung der Zeit, für die eine Aussage gemacht wird. Klein (1994) hat hierfür die Bezeichnung Topikzeit geprägt. In unserem Fall (52) dient das Lokaladverbial also zur Ausgliederung der Topikzeit, für die die Aussage Gültigkeit beansprucht. (52) macht damit eine Aussage über Luise zu einer Zeit, als sie in Portugal war, nämlich daß sie zu dieser Zeit reich war. Wir haben es also mit zwei Zeiten zu tun, der durch Rahmenadverbial und Tempus ausgegliederten Topikzeit (kurz: $\mathrm{t}^{\mathrm{T}}$; in (53) notiert als " $x \times \times x^{\text {") }}$ sowie der durch das VPDenotat gegebenen Kommentarzeit ${ }^{21}$ (kurz: ${ }^{\mathrm{C}}$; in (53) notiert als "/I//"). ${ }^{22}$ Die Grammatik läßt offen, ob (a) diese beiden Zeiten deckungsgleich sind $\left(\mathrm{t}^{\mathrm{T}}=\mathrm{t}^{\mathrm{C}}\right)$, ob (b) Luise auch vor und/oder nach ihrem Portugal-Aufenthalt reich war $\left(\mathrm{t}^{\mathrm{T}} \subset \mathrm{t}^{\mathrm{C}}\right)$, ob (c) sie etwa ihr ganzes Leben lang reich war, oder (d) Portugal möglicherweise nie verlassen hat, sprich: ob wir es hier mit permanenten Eigenschaften von Luise zu tun haben. In diesem Fall würde $\mathrm{t}^{\mathrm{T}}$ und/oder $\mathrm{t}^{\mathrm{C}}$ (vereinfacht gesagt) mit Luises Lebenszeit (kurz: $\mathrm{t}^{\mathrm{L}}$; in (53) notiert als "»m»»") übereinstimmen. In (53) sind die relevanten Zeitverhältnisse schematisch angegeben. ${ }^{23}$

\footnotetext{
21 Klein (1994) verwendet hierfür den Begriff der Situationszeit. Ich bevorzuge die Redeweise von einer Kommentarzeit aus zwei Gründen: Erstens haben die vorangegangenen Überlegungen gezeigt, daß nicht alle VPs Situationen bezeichnen. Zweitens verdeutlicht diese Namensgebung über die temporalen und aspektuellen Bezüge hinaus den Zusammenhang mit der Topik/Kommentar-Gliederung des Satzes; s. hierzu den Vorschlag für eine kompositionale Semantik von TopP in Maienborn (1998).

22 Im Falle von Kopula-Prädikativ-Konstruktionen und stativen Verben liegt $\mathrm{t}^{\mathrm{C}}$ auf VP-Ebene unmittelbar vor; s. (50.b). Bei situationsdenotierenden VPs ist $t^{C}$ durch die Situationsdauer gegeben.

23 Die Angaben in (53) sind in mehrerlei Hinsicht eine Idealisierung. Damit cine Eigenschaft als permanent wahrgenommen wird, muß sie sich nicht notwendigerweise bereits bei der Geburt einstellen. Wichtig ist vielmehr, daß es ab dem Zeitpunkt ihres Auftretens keine Zeiten mehr gibt, zu denen sie nicht vorliegt. Dies ist z.B. bei den Fällen in (i) zu ganz unterschiedlichen Zeiten gegeben.

(i) Luise ist Portugiesin / Katholikin / Vegetarierin / Gewerkschafterin.

Für die hier vorgetragene Argumentation können wir diese Komplikationen vernachlässigen. Ebenso werde ich hier den Beitrag des Tempus für die Bestimmung der Topikzeit ausblenden, sprich: die Einordnung von $\mathrm{t}^{\mathrm{T}}$ relativ zur Äußerungszeit; s. Klein (1994). Zu den bei präteritalen Sätzen zu beobachtcnden sog. "lifetime effects" s. die pragmatische Erklärung von Musan (1995, 1997).
} 

a. $\mathrm{t}^{\mathrm{T}}$ :
$x \times x \times x \times x \times x \times x \times x \times x \times x$
$t^{C}$ :
IIIIIIIIIIIIIIIIIIIIIIIIIIIIIIIIIIII
$\mathrm{t}^{\mathrm{T}}=\mathrm{t}^{\mathrm{C}}$
$\mathrm{t}^{\mathrm{L}}$.

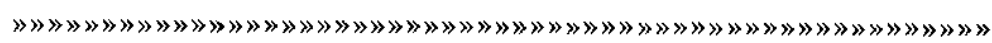
$\mathrm{t}^{\mathrm{C}} \subset \mathrm{t}^{\mathrm{L}}$
b. $\mathrm{t}^{\mathrm{T}}$ :
tC:
$x \times x \times x \times x \times x \times x$
$\mathrm{L}$ :

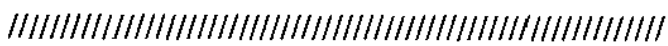
$\mathrm{t}^{\mathrm{T}} \subset \mathrm{t}^{\mathrm{C}}$

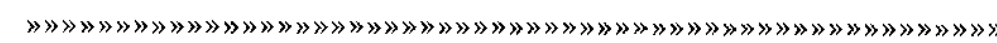
$t^{\mathrm{C}} \subset \mathrm{t}^{\mathrm{L}}$
c. $\mathrm{t}^{\mathrm{T}}$ :

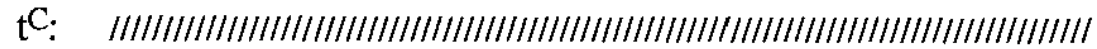
$\mathrm{t}^{\mathrm{T}} \subset \mathrm{t}^{\mathrm{C}}$

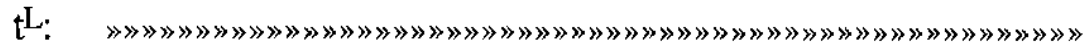
$\mathrm{t}^{\mathrm{C}}=\mathrm{t}^{\mathrm{L}}$
d. $\mathrm{t}^{\mathrm{T}:} \quad x \times x \times x \times x \times x \times x \times x \times x \times x \times x \times x \times x \times x \times x \times x \times x \times x \times x \times x \times x \times x \times x \times x \times x \times x \times x$

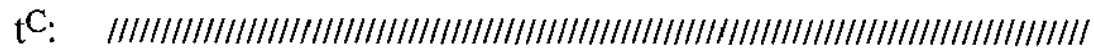

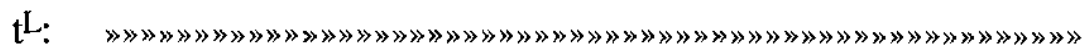
$\mathrm{t}^{\mathrm{T}}=\mathrm{t}^{\mathrm{C}}$
$\mathrm{t}^{\mathrm{C}}=\mathrm{t}^{\mathrm{L}}$

Die Grammatik verhält sich also gegenüber diesen Optionen neutral, pragmatisch sind sie durchaus unterschiedlich gewichtet. Ich will die hier anzutreffenden pragmatischen Bewertungen auf der Grundlage von konversationellen Implikaturen rekonstruieren, Schlußfolgerungen also, die vor dem Hintergrund der Griceschen (1975) Konversationsmaximen gezogen werden. Für die weiteren Überlegungen werde ich die bei Atlas \& Levinson (1981) und Horn (1984) eingeführte Reduzierung der Konversationsmaximen auf zwei grundlegende Prinzipien, dem Q-Prinzip und dem I-Prinzip (bei Horn: R-Prinzip), zugrundelegen; s. auch Levinson (1998) sowie die Weiterentwicklung und Formalisierung in Blutner (1999). In (54) sind die beiden Prinzipien in den Formulierungen von Horn (1984) und Levinson (1998) angegeben.

(54) a. Q-Prinzip: Say as much as you can (given I).

Horn (1984: 13)

Select the informationally strongest paradigmatic alternate which is consistent with the facts.

Levinson (1998: 102)

b. I-Prinzip: Say no more than you must (given Q).

Horn (1984: 13)

Produce the minimal linguistic information sufficient to achieve your communicational ends (bearing $Q$ in mind.)

Levinson (1998: 156)

Ein Sprecher, der das Q-Prinzip befolgt, wird seinen Beitrag so informativ wie möglich gestalten. Wenn er die Wahl zwischen alternativen Äußerungen von vergleichbarer sprachlicher Komplexität hat, wird er die stärkstmögliche Aussage auswählen. Dies versetzt den Hörer in die Lage zu schlußfolgern, daß stärkere Aussagen als die vom Sprecher gewählte offenbar nicht zutreffen. Zu den Q-basierten Implikaturen gehören insbesondere skalare Implikaturen; s. z.B. die Sammlung in Levinson (1998: 103ff). Das I-Prinzip erlaubt es dem Hörer demgegenüber, naheliegende Information zu ergänzen, beispielsweise vom allgemeinen Fall auf eine typische Instanz zu schließen, um so zu einer spezifischen Interpretation im jeweiligen Äußerungskontext zu gelangen. $\mathrm{Zu}$ den I-basierten Implikaturen gehören etwa die Deutung der Konjunktion bei Ereignissen als temporale oder kausale Folge oder die Verengung der Interpretation einer konditionalen Aussage auf das Bikonditional (sog. "Conditional Perfection"; s. Geis \& Zwicky 1971); s. auch hier die Aufstellung in Levinson (1998: $159 \mathrm{ff})$.

Betrachten wir vor diesem Hintergrund die in (53) schematisch wiedergegebenen Spezifizierungsoptionen der temporalen Lesart eines Satzes wie (52). Zunächst können wir den Fall, in dem das Lokaladverbial eine permanente Eigenschaft bezeichnet $-\mathrm{t}^{\mathrm{T}}$ und $\mathrm{t}^{\mathrm{L}}$ also zusammenfallen; $\mathrm{s}$. (53.d)) als Verletzung des Q-Prinzips ausschließen. Hier trägt der rahmensetzende Modifikator nichts zum Einschränkung der Topikzeit und damit zum Informationsgehalt der Aussage bei. Der Sachverhalt läßt 
sich auch ohne rahmensetzenden Modifikator, spachlich also weniger aufwendig, ausdrücken; s. (55).

(55) Luise war reich.

Aus der Tatsache, daß sich der Sprecher für die sprachlich aufwendigere Formulierung (52) entschieden hat, kann der Hörer auf der Basis des Q-Prinzips schließen, daß die Interpretationsvariante (53.d), für die es mit (55) eine einfachere Ausdrucksmöglichkeit gibt, nicht vom Sprecher intendiert ist. $\mathrm{Daß}$ es sich hierbei um eine konversationelle Implikatur handelt, zeigt die Variante (52'). Hier wird die Q-basierte Implikatur durch den Kontext explizit aufgehoben. (52') ist folglich kompatibel mit den in (53.d) angegebenen Zeitverhältnissen.

(52') Stell Dir mal vor, was geschehen wäre, wenn Luise in Monaco gelebt hätte. Sie hätte doch im $\mathrm{Nu}$ ihr ganzes Geld im Casino verspielt. Solche Versuchungen boten sich in Portugal zum Glück nicht. Deswegen war Luise in Portugal reich. Aufhebung der Q-basierten Implikatur

Halten wir fest: Die temporale Interpretation rahmensetzender Modifikatoren ist pragmatisch nur dann zulässig, wenn die Topikzeit durch den Modifikator echt beschränkt wird. Sofern die Q-basierte Implikatur nicht explizit außer Kraft gesetzt ist, dürfen wir im Falle von Lokaladverbialen davon ausgehen, daß die angegebene Lokalisierung nur zeitweilig vorliegt, daß wir es also mit einer temporären Eigenschaft des betreffenden Individuums zu tun haben. Dies setzt entweder die Mobilität des Subjektreferenten oder aber eine in das Rahmenadverbial eingebaute zeitliche Befristung voraus.

Zur Mobilität des Subjektreferenten: Bewegliche Individuen können ihren Ort verändern und lassen damit eine temporäre Lokalisierung zu. Ist die Forderung nach Mobilität nicht mit unserem Weltwissen vereinbar (und sind wir nicht bereit, die präsupponierte Ortsveränderung in irgendeiner Weise zu akkomodieren), so scheidet die temporale Lesart als Interpretationsvariante für den rahmensetzenden Modifikator aus. Dies trifft z.B. auf (56) zu. Wenn wir darauf beharren, daß sich das Brandenburger Tor seit seiner Erbauung nicht vom Fleck gerührt hat, können wir den Satz nicht als Aussage über eben jenes Tor zur Zeit, als es sich in Berlin befand, auffassen.

(56) In Berlin war das Brandenburger Tor für Pferdekutschen gesperrt.

keine temporale Lesart

Zur eingebauten zeitlichen Befristung beim Rahmenadverbial: Unabhängig von der Mobilität der zu lokalisierenden Entität liegt eine temporäre Lokalisierung auch dann vor, wenn das interne Argument der Präposition ein lokales Referenzobjekt bezeichnet, das (a) seinerseits ortsveränderlich ist wie z.B. in (57.a), oder (b) nur zeitlich befristet existiert wie in (57.b/c).

(57) a. Unter der Plane war das Brandenburger Tor vor Regen und Schnee geschützt.

b. In der Sonne / Im Mondlicht war das Brandenburger Tor ein beliebtes Photomotiv.

c. In Preußen / In der DDR war das Brandenburger Tor für den Verkehr gesperrt.

In den Sätzen unter (57) drückt das Lokaladverbial eine räumliche Lokalisierung aus, die wir aufgrund unseres Weltwissens unabhängig von der zu lokalisierenden Entität als zeitlich begrenzt auffassen können. Infolgedessen ist hier auch bei ortsunveränderlichen Subjektreferenten die temporale Deutung des rahmensetzenden Modifikators möglich.

Soviel zur pragmatischen Beschränkung temporal gedeuteter rahmensetzender Modifikatoren auf temporäre Eigenschaften. Unter den verbleibenden Spezifizierungsoptionen (53.a-c) für die temporale Interpretation eines rahmensetzenden Modifikators bei Kopula-Prädikativ-Konstruktionen nimmt die Variante (53.a) eine Sonderstellung ein. Sie stellt den typischen Fall dar und erfüllt somit neben dem Q-Prinzip auch das I-Prinzip. Die Interpretation, daß Luise zur Zeit ihres Portugal-Aufenthalts reich war, wird pragmatisch verengt zu der Annahme, daß Luise zu genau dieser Zeit reich war, nicht unmittelbar davor und nicht unmittelbar danach. Diese I-basierte Implikatur folgt dem Muster der "conditional perfection"; s.o. Das heißt, das semantisch unterbestimmte Verhältnis zwischen Topikzeit und Kommentarzeit $\mathrm{t}^{\mathrm{T}} \subseteq \mathrm{t}^{\mathrm{C}}$ wird pragmatisch als Identität spezifiziert. 
Auch in diesem Fall kann die konversationelle Implikatur explizit aufgehoben werden. In einem Kontext beispielsweise, in dem Ungewißheit über den finanziellen Hintergrund Luises besteht, kann der Sprecher mit der Äußerung von (52) ausdrücken, daß er in Luises Verhalten während ihres Portugal-Aufenthalts einen Hinweis darauf sieht, daß Luise in der Tat ein Leben in Reichtum führt; s. $\left(52^{\prime \prime}\right)$. Die Variante $\left(52^{\prime \prime}\right)$ ist folglich kompatibel mit den in $(53 . \mathrm{b} / \mathrm{c})$ angegebenen Zeitverhältnissen.

(52") Wir wissen doch inzwischen, daß entweder Luise oder Heidi unsere gesuchte Millionärin ist, und ihr habt alle mitgekriegt, daß Luise in Portugal reich war. Ich tippe also auf Luise.

Aufhebung der I-basierten Implikatur

Fassen wir zusammen. Die Vorgaben für die temporale Interpretation rahmensetzender Modifikatoren bei Kopula-Prädikativ-Konstruktionen sind in (58.a) angegeben: Die Topikzeit ist als unechtes Teilintervall der Lebenszeit des Subjektreferenten ausgewiesen, sowie als unechtes Teilintervall der Kommentarzeit. Die Festlegung der Topikzeit als echtes Teilintervall der Lebenszeit des Subjektreferenten ist Ergebnis einer Q-basierten Implikatur und beruht auf dem Vergleich mit alternativen Ausdrucksmöglichkeiten; s. (58.b). Die Identifikation von Topikzeit und Kommentarzeit resultiert aus einer I-basierten Implikatur und beruht auf dem Vergleich mit alternativen Interpretationsoptionen; $s$. (58.c). ${ }^{24}$

(58) Temporale Interpretation rahmensetzender Modifikatoren bei Kopula-Prädikativ-Konstruktionen:
a. Ausgangslage:
$\mathrm{t}^{\mathrm{T}} \subseteq \mathrm{t}^{\mathrm{L}} \& \mathrm{t}^{\mathrm{T}} \subseteq \mathrm{t}^{\mathrm{C}}$
b. Q-basierte Implikatur: $\mathrm{t}^{\mathrm{T}} \subset \mathrm{t}^{\mathrm{L}}$
c. I-basierte Implikatur: $\quad \mathbf{t}^{\mathrm{T}}=\mathrm{t}^{\mathrm{C}}$

Wenn nun der Beitrag eines rahmensetzenden Modifikators zur Beschränkung der Topikzeit pragmatisch nur dann zulässig ist, wenn es sich um eine temporäre Eigenschaft handelt, und aus unabhängigen Gründen die durch die Kopula-Prädikativ-Konstruktion ausgewiesene Kommentarzeit bevorzugt mit der Topikzeit identifiziert wird, so folgt daraus, daß die von dem Prädikativ bezeichnete Eigenschaft ebenfalls als temporär eingestuft wird. Dies ist die Grundkonstellation bei den hier betrachteten Kopula-Prädikativ-Konstruktionen mit temporal gedeuteten rahmensetzenden Modifikatoren: Die Hörer erwarten, daß sich die durch das Prädikativ bezeichnete Eigenschaft über genau den durch den rahmensetzenden Modifikator ausgegliederten Zeitraum erstreckt. (Immer vorausgesetzt, daß die konversationellen Implikaturen nicht explizit unterbunden werden.)

Die beobachteten Akzeptabilitätsunterschiede beziehen sich auf die Plausibilität, die wir dieser Synchronisation zweier Eigenschaften vor dem Hintergrund unseres Kontext- und Weltwissens beimessen. Betrachten wir hierzu die Sätze unter (59):

In Portugal war Luise

a. glücklich/fröhlich/sympathisch.

b. reich/verheiratet/braungebrannt/ausgeglichen/Gewerkschafterin.

c. müde/hungrig/betrunken/ohnmächtig.

d. Linkshänderin/intelligent.

e. tot/Witwe/Nachkomme Bismarcks/Sternzeichen Fische.

keine temporale Lesart

Was die durch das Rahmenadverbial in Portugal ausgegliederte Zeitphase anbelangt, so sagt uns unser Weltwissen, daß sich der Aufenthalt in einem Land in der Regel nicht im Stundenbereich abspielt. Darüber hinaus haben wir keine besonderen Erwartungen an die Aufenthaltsdauer. Mit den in (59.a) angegebenen Eigenschaften verbinden sich keine besonderen Annahmen über ihre typische

\footnotetext{
24 S. Levinson (1998: 162f) und Blutner (1999) zu der unterschiedlichen Ausrichtung konversationeller Implikaturen an alternativen Ausdrucksformen für einen Bedeutungsgehalt im Falle des Q-Prinzips und alternativen Interpretationsoptionen eines sprachlichen Ausdrucks im Falle des I-Prinzips.
} 
Dauer. Glücklich-Sein kann eine Sache von Sekunden oder eines ganzen Lebens sein. Der pragmatisch herbeigeführten zeitlichen Befristung und Synchronisation dieser beiden Eigenschaften steht damit nichts im Wege. Die Prädikative unter (59.b) bezeichnen Eigenschaften, die sich in der Regel über eine etwas längere Zeitphase erstrecken. Die erschlossene Mindestdauer des PortugalAufenthalts ist deshalb bei (59.b) länger als bei (59.a).

Im Falle der unter (59.c) aufgeführten Prädikative haben wir es demgegenüber mit Eigenschaften zu tun, die typischerweise nur kurzzeitig auftreten. Um diese Eigenschaften mit der durch das Rahmenadverbial bezeichneten Lokalisierungseigenschaft zeitlich in Einklang zu bringen, können wir entweder versuchen, den Beitrag des Rahmenadverbials an die zeitlichen Vorgaben des Prädikativs anzupassen, indem wir beispielsweise annehmen, daß es sich bei dem Aufenthalt in Portugal lediglich um eine kurze Zwischenlandung mit dem Flugzeug handelt, oder umgekehrt das Prädikativ dem Rahmenadverbial angleichen, indem wir von einer Iteration der betreffenden Eigenschaften ausgehen, die es gestattet, den Aufenthalt in Portugal als eine Zeitphase darzustellen, in der Luise charakteristischerweise oder immerzu müde/hungrig usw. war.

Die Prädikative in (59.d) und (59.e) schließlich bezeichnen Dispositionen bzw. irreversible Eigenschaften. Bei ersteren ist eine zeitliche Befristung nur sehr schwer mit unserem Weltwissen vereinbar, bei letzteren ist dies von vorneherein ausgeschlossen. Folglich sperren sich diese Prädikative gegen eine temporale Rahmensetzung. In (59.d) ist die temporale Interpretation des rahmensetzenden Modifikators nur unter sehr aufwendigen, im jeweiligen Kontext zu rechtfertigenden Annahmen über die Veränderung von Dispositionen möglich. Für (59.e) ist dies nicht gegeben. Es gibt keine Möglichkeit, eine irreversible Eigenschaft als temporär auszuweisen, also scheidet die temporale Lesart des rahmensetzenden Modifikators bei (59.e) aus. (Es verbleiben die epstemische Deutung und ggf. weitere Interpretationsmöglichkeiten rahmensetzender Modifikatoren.)

Wenn wir den rahmensetzenden Modifikator nun wie in (60) variieren, d.h. ein Lokaladverbial wählen, mit dem wir typischerweise eine kürzere Verweildauer der zu lokalisierenden Entität an dem angegebenen Ort assoziieren, so verlagert sich der Legitimationsdruck an den Kontext: (60.c) bedarf nun keinerlei zusätzlicher Annahmen mehr, um die temporale Interpretation zu rechtfertigen. (60.b) hingegen ist auf mehr oder weniger aufwendige kontextuelle Zusatzannahmen angewiesen, um ein zeitliches Zusammenfallen der betreffenden Eigenschaften zu motivieren. Und die Aussichten, für (60.d) eine plausible Legitimation für die temporale Interpretation zu finden, sinken gegenüber (59.d) noch einmal beträchtlich. Im Falle der zeitlich nicht festgelegten Eigenschaften in (60.a) sowie der irreversiblen Eigenschaften (60.e) ändert sich nichts gegenüber den entsprechenden Konstellationen in (59).

$\left\{\begin{array}{l}\text { Im Auto } \\ \text { Unter der Brücke } \\ \text { Auf dem Heimweg }\end{array}\right\}$ war Luise

I a. glücklich/fröhlich/sympathisch.

b. reich/verheiratet/Gewerkschafterin.

c. müde/hungrig/betrunken/ohnmächtig.

d. Linkshänderin/intelligent.

e. tot/Witwe/Sternzeichen Fische. keine temporale Lesart

Die hier vorgeschlagene Analyse läßt sich in den wesentlichen Punkten auf den Fall der temporalen Modifikatoren übertragen; s. die Beispiele (27) - (29) in Abschnitt 2.1. Hier bedarf es keines Umweges über die temporäre Lokalisierung im Raum, um die Topikzeit auszugliedern, sondern diese kann durch den temporalen Modifikator direkt beschränkt werden. (Unterschiede ergeben sich allerdings durch die vielfältigeren Möglichkeiten der grammatischen Integration temporaler Modifikatoren im Bereich der IP.) Die Akzeptabilitätsschwankungen reflektieren auch hier die Kompatibilität der Topikzeit mit unseren Annahmen über die typische bzw. mögliche Dauer der im Kommentar ausgedrückten Eigenschaft. 
Soweit eine pragmatische Erklärung der Akzeptabilitätsunterschiede bei Kopula-Prädikativ-Konstruktionen mit rahmensetzenden Modifikatoren. Sie beruht auf der Wirksamkeit konversationeller Implikaturen und nutzt Weltwissen über die mögliche zeitliche Erstreckung von Eigenschaften. Nach der hier vertretenen Auffassung orientiert sich die Akzeptabilität entsprechender Konstruktionen an der Kompatibilität der von Prädikativ und Rahmenadverbial bezeichneten Eigenschaften hinsichtlich ihrer temporalen Erstreckung. Die Befristung und zeitliche Gleichsetzung der betreffenden Eigenschaften ist dabei nicht grammatisch angelegt, sondern wird pragmatisch induziert.

Die hier vorgeschlagene Analyse folgt nicht der in Abschnitt 1.2 wiedergegebenen Annahme Chierchias (1995), für (eine Teilklasse von) Eigenschaften eine räumliche Situierung vorzusehen. Die durch das Rahmenadverbial beigesteuerte Lokalisierung im Raum und die vom Prädikativ bezeichnete Eigenschaft bestehen vielmehr unabhängig voneinander. Es ist lediglich die Lokalisierung in der Zeit, die diese beiden Eigenschaften miteinander teilen. Die lokale Rahmensetzung dient dazu, eine bestimmte Zeitphase auszugliedern, für die die vom Prädikativ bezeichnete Eigenschaft auf das Subjekt zutrifft.

In der Frage der Stadien/Individuen-Distinktion kommt die vorliegende Untersuchung zu dem Ergebnis, daß eine Unterscheidung temporärer vs. permanenter Eigenschaften weder grammatisch noch begrifflich eigens ausgewiesen ist. Hinweise auf grammatische Reflexe wurden in den vorangehenden Abschnitten entkräftet. Was die begriffliche Seite anbelangt, so zeigen die Überlegungen in diesem Abschnitt, daß die temporale Interpretation rahmensetzender Modifikatoren bei KopulaPrädikativ-Konstruktionen auf feineren und flexibleren Einteilungen von Eigenschaften nach ihrer typischen bzw. möglichen zeitlichen Erstreckung beruht. Dabei kommt allenfalls den irreversiblen Eigenschaften sowie ggf. Dispositionen ein besonderer, vorab zu vermerkender Status zu. In den übrigen Fällen ist die Frage "permanent oder temporär?" nicht unabhängig vom Träger der Eigenschaft in einem konkreten Kontext zu entscheiden und liefert damit kein sinnvolles Kriterium für eine stabile, lexikalisch verankerte Klassifikation von Eigenschaften.

Folgt man den hier vorgetragenen Überlegungen zum Situationsbezug und zur Stadien/IndividuenDistinktion bei Kopula-Prädikativ-Konstruktionen, so ist nicht nur eine gehörige Portion Skepsis bei der gegenwärtig zu beobachtenden inflationären Beanspruchung von Situationen, Ereignissen und dergleichen angebracht, sondern die Verpflichtung auf möglichst rigide grammatische und ontologische Annahmen für die Kategorie der Situationen liefert zugleich auch den Schlüssel zu einem genaueren Verständnis des Zusammenspiels von Grammatik und Pragmatik bei der Bedeutungskonstitution.

\section{Literatur}

Atlas, J. \& S. Levinson (1981): It-Clefts, Informativeness and Logical Form. In: P. Cole (ed.), Radical Pragmatics, New York: Academic Press, $1-61$.

Bach, Emmon (1986): The algebra of events. Linguistics and Philosophy 9: 5 - 16.

Bach, Emmon (1989): Informal Lectures on Formal Semantics. New York: State University of New York Press.

Bayer, Josef (1986): The role of event expressions in grammar. Studies in Language 10/1: 1 - 52.

Bierwisch, Manfred (1982): Formal and Lexical Semantics. Linguistische Berichte 80/82, 3 - 17.

Bierwisch, Manfred (1983): Semantische und konzeptuelle Repräsentationen lexikalischer Einheiten. In: R. Ruzicka \& W. Motsch (eds.), Untersuchungen zur Semantik (= studia grammatica XXII). Berlin: Akademie-Verlag, $61-99$.

Bierwisch, Manfred (1988): On the Grammar of Local Prepositions. In: M. Bierwisch, W. Motsch \& I. Zimmermann (eds.), Syntax, Semantik und Lexikon, Berlin: Akademie-Verlag, $1-65$.

Blutner, Reinhard (1999): Lexical Pragmatics. Journal of Semantics 15/2: 115 - 162.

Carlson, Greg (1978): Reference to Kinds in English. Ph.D. Dissertation, University of Massachusetts, Amherst. 
Chierchia, Gennaro (1995): Individual-Level Predicates as Inherent Generics. In: G. N. Carlson \& F. J. Pelletier (eds.), The Generic Book. Chicago/London: The University of Chicago Press, $176-223$.

Davidson, Donald (1967): The Logical Form of Action Sentences. In: D. Davidson (1980), Essays on Actions and Events. Oxford: Clarendon Press, $105-122$.

Diesing, Molly (1992): Indefinites. Cambridge, Mass.: The MIT Press.

Diesing, Molly (1992a): Bare Plural Subjects and the Derivation of Logical Representations. Linguistic Inquiry 23/3: $353-380$.

Dölling, Johannes (1997): Ist die Kopula mehrdeutig? Anmerkungen zu einem Vorurteil. In: U. Scheffler \& K. Wuttich (eds.), Terminigebrauch und Folgebeziehung. Logos: Berlin, 5-24.

Dowty, David R. (1979): Word Meaning and Montague Grammar. Dordrecht: Reidel.

Eckardt, Regine (1998): Adverbs, Events and Other Things. Issues in the Semantics of Manner Adverbs (= Linguistische Arbeiten 379). Tübingen: Niemeyer.

Egg, Markus (1994): Aktionsart und Kompositionalität (= studia grammatica XXXVII), Berlin: Akademie-Verlag.

Ehrich,Veronika (1991): Nominalisierungen. In: A. v. Stechow \& D. Wunderlich (eds.), Semantik - Semantics: Ein internationales Handbuch der zeitgenössischen Forschung - An International Handbook of Contemporary Research. Berlin/New York: de Gruyter, 441 - 458.

Geis, M. \& A. Zwicky (1971): On invited inferences. Linguistic Inquiry 2: 561 - 565.

Geist, Ljudmila (1998): Kopulaverben und Prädikativkonstruktionen: Ein deutsch-russischer Vergleich. Magisterarbeit Humboldt-Universität Berlin.

Geist, Ljudmila (1999): Russisch byt' ('sein') als funktionale und/oder lexikalische Kategorie. In diesem Band.

Grice, H. P. (1975): Logic and Conversation. In: P. Cole \& J. L. Morgan (eds.), Syntax and Semantics 3: Speech Acts. New York: Academic Press, 41 - 58.

Heim, Irene (1982): The Semantics of Definite and Indefinite Noun Phrases. Ph.D. Dissertation, University of Massachusetts, Amherst.

Heim, Irene \& Angelika Kratzer (1998): Semantics in Generative Grammar. Malden/Oxford: Blackwell.

Herweg, Michael (1990): Zeitaspekte. Die Bedeutung von Tempus, Aspekt und temporalen Konjunktionen. Wiesbaden: Deutscher Universitäts-Verlag.

Higginbotham, James (1985): On Semantics. Linguistic Inquiry 16, $547-593$.

Higginbotham, James (1983): The Logic of Perceptual Reports: An Extensional Alternative to Situation Semantics. Journal of Philosophy LXXX/2: 100 - 127.

Higginbotham, James (1994): The Semantics and Syntax of Event Reference. ESSLLI'94 Course Notes.

Higginbotham, James \& Gillian Ramchand (1996): The Stage-Level/Individual-Level Distinction and the Mapping Hypothesis. Manuscript, University of Oxford.

Horn, L.R. (1984): Towards a New Taxonomy for Pragmatic Inference: Q-Based and R-Based Implicatures. In: D. Schiffrin (ed.), Meaning, Form, and Use in Context. Washington: Georgetown University Press, $11-42$.

Jacobs, Joachim (1995): Wieviel Syntax braucht die Semantik? Möglichkeiten und Grenzen einer sparsamen Theorie der Bedeutungskomposition. Arbeiten des SFB 282 "Theorie des Lexikons" Nr. 73. Universität Wuppertal.

Jäger, Gerhard (1997): The Stage/Individual Contrast Revisited. In: Brian Agbayani \& Sze-Wing Tang, Proceedings of WCCFL 15. Stanford: CSLI, $225-239$

Jäger, Gerhard (1999): Stage levels, states, and the semantics of the copula. In diesem Band.

Kamp, Hans (1981): A Theory of Truth and Semantic Representation. In: J. Groenendijk \& T. Janssen \& M. Stokhof (eds.), Formal Methods in the Study of Language. Mathematisch Centrum: Amsterdam, $277-322$.

Kamp, Hans \& Reyle, Uwe (1993): From Discourse to Logic. Introduction to Modeltheoretic Semantics of Natural Language, Formal Logic and Discourse Representation Theory. Dordrecht/Boston/London: Kluwer.

Klein, Wolfgang (1994): Time in Language. London/New York: Routledge.

Kratzer, Angelika (1989): Stage-Level and Individual-Level Predicates. Published in: G. N. Carlson \& F. J. Pelletier (1995, eds.), The Generic Book. Chicago/London: The University of Chicago Press, 125 - 175.

Krifka et al. (1995): Genericity: An Introduction. In: G. N. Carlson \& F. J. Pelletier (eds.), The Generic Book. Chicago/London: The University of Chicago Press, $1-124$.

Levinson, Stephen C. (1998): Presumptive Meanings: The Theory of Generalized Conversational Implicature. Manuscript, MPI for Psycholinguistics, Nijmegen.

Lewis, David (1975): Adverbs of Quantification. In: E. Keenan (ed.), Formal Semantics of Natural Language. Cambridge: Cambridge University Press, $3-15$. 
Maienborn, Claudia (1996): Situation und Lokation: Die Bedeutung lokaler Adjunkte von Verbalprojektionen (= Studien zur deutschen Grammatik 53), Tübingen: Stauffenburg.

Maienborn, Claudia (1998): The Grammar and Pragmatics of Locative Modifiers. Manuscript, Humboldt-Universität Berlin.

Mourelatos, Alexander P. D. (1978): Events, Processes, and States. Linguistics and Philosophy 2: 415 - 434.

Musan, Renate (1995): On the Temporal Interpretation of Noun Phrases. Ph.D. dissertation, MIT.

Musan, Renate (1997): Tense, Predicates, and Lifetime Effects. Natural Language Semantics 5/3: 271 - 301.

Musan, Renate (1999): Zur Semantik von werden. Ist prädikatives werden transitional? In diesem Band.

Parsons, Terence (1990): Events in the Semantics of English. A Study in Subatomic Semantics. Cambridge, Mass: The MIT Press.

Parsons, Terence (1995): Thematic Relations and Arguments. Linguistic Inquiry 26/4: 635 - 662.

Schmitt, Cristina (1992): ser and estar: A Matter of Aspect. Proceedings of NELS 22: 411 - 426.

Schmitt, Cristina (1999): When STAY and BECOME are the same verb: the case of ficar. In diesem Band.

Steinitz, Renate (1999): Die Kopula werden und die Situationstypen. In diesem Band.

Steinitz, Renate (1999a): Deutsch werden, bleiben: Schwedisch bli, förbli. In diesem Band.

Urbas, Martina (1993): Numeruskongruenz und Numeruskonflikte in Kopulasätzen. Arbeiten des SFB 282 "Theorie des Lexikons" Nr. 38. Universität Düsseldorf.

Vendler, Zeno (1967): Linguistics in Philosopy. Ithaca, New York: Cornell University Press.

Zimmermann, Ilse (1992): Der Skopus von Modifikatoren. In: I. Zimmermann \& A. Strigin (eds.), Fügungspotenzen (= studia grammatica 34), Berlin: Akademie-Verlag, $251-279$.

Zimmermann, Ilse (1998): Die Integration topikalischer DPs in die syntaktische und semantische Struktur von Sätzen. Erscheint in: M. Doherty (ed.), Sprachspezifische Aspekte der Informationsverteilung (= studia grammatica), Berlin: Akademie-Verlag.

Zimmermann, Ilse (1999): Partizip II-Konstruktionen des Deutschen als Modifikatoren. In diesem Band. 